\title{
Financial reporting and market efficiency with extrapolative investors*
}

\author{
Milo Bianchi ${ }^{\dagger} \quad$ Philippe Jehiel ${ }^{\ddagger}$
}

February 16, 2015

\begin{abstract}
We model a financial market in which companies engage in strategic financial reporting knowing that investors only pay attention to a randomly drawn sample from firms' reports and extrapolate from this sample. We investigate the extent to which stock prices differ from the fundamental values, assuming that companies must report all their activities but are otherwise free to disaggregate their reports as they wish. We show that no matter how large the samples considered by investors are, a monopolist can induce a price of its stock bounded away from the fundamental. Besides, increasing the number of companies competing to attract investors may exacerbate the mispricing of stocks.
\end{abstract}

Keywords: Extrapolation, efficient market hypothesis, competition, sophistication, financial reporting.

JEL codes: C72, D53, G14.

${ }^{*}$ We thank Alessandro Pavan (editor) and three referees for constructive comments. We also thank participants at the conference on Finance and Expectational Coordination at NYU (especially the discussant, Stephen Morris), the NBER Behavioral Economics working group Fall 2012 (especially the discussant, Brett Green), the workshop on Bounded Rationality, Jerusalem 2012, Warwick Creta workshop 2012, and various seminar participants. Milo Bianchi thanks the Risk Foundation (Groupama Chair "Les Particuliers Face aux Risques" and SCOR/IDEI Chair "Market Risk and Value Creation") and Philippe Jehiel thanks the European Research Council for financial support.

${ }^{\dagger}$ Corresponding author. Toulouse School of Economics. 21, allée de Brienne 31000 - Toulouse (France). E-mail: milo.bianchi@tse-fr.eu. Phone: +33 567732759.

${ }^{\ddagger}$ Paris School of Economics, Ecole des Ponts, Paritech and University College London. E-mail: jehiel@enpc.fr 


\section{Introduction}

The recent financial crisis as well as some famous accounting scandals have revealed that some firms can deliberately obfuscate their financial statements, and that many investors may lack the sophistication needed to read through such opaqueness. As a result, financial markets may not be efficient in that stock prices may be far from the underlying fundamentals. A typical regulatory response would be to impose tighter disclosure requirements on firms while at the same time attempting to "educate" investors, if possible. ${ }^{1}$ Another kind of response may instead rely on market forces, hoping that the competition to attract investors would discipline firms and lead to market efficiency.

In this paper, we develop a simple framework to investigate the impact of strategic financial reporting on whether the prices of stocks correctly reflect fundamental values. We focus on a setting in which investors are not fully sophisticated in the way they interpret the information provided by firms, and at the same time firms are required to meet (strong) regulatory standards insofar that all activities in the firm have to be referred to in the financial report. We analyze how firms' reporting strategies and market prices vary as investors' degrees of sophistication vary and/or as more firms compete to attract investors.

Specifically, we consider a stylized financial market in which each firm simultaneously chooses a financial report with the objective of influencing investors' beliefs and ultimately maximizing the trading price on the stock market. ${ }^{2} \mathrm{~A}$ report consists in a set of signals about the firm's profitability (how much investors can expect to receive for each dollar invested in the firm). We assume that each firm is constrained to choose a set of signals whose mean corresponds to the true profitability, while at the same time being able to freely affect the noise in the signals' distribution.

Such a report can be viewed as a statement about the profitability of the firm. The firm can choose to make a very simple statement, a single number summarizing the overall profitability of the firm, or a more complicated statement,

\footnotetext{
${ }^{1}$ Forms of investor protection aimed at enhancing the reliability of financial reports were famously advocated by SEC Chairman Arthur Levitt (Levitt (1998)), and then incorporated in the Regulation for Fair Disclosure. Increasing the transparency of corporate disclosures lies at the heart of recent interventions such as Sarbanes-Oxley Act (adopted after Enron) and DoddFrank Reform (adopted after the subprime crisis). The latter has also created the Consumer Financial Protection Bureau with the intent of improving investors' sophistication.

${ }^{2}$ Managers' compensation is directly influenced by trading prices through stock options say. Evidence suggests a strong link between performance-related compensation and aggressive accounting practices, see Burns and Kedia (2006); Bergstresser and Philippon (2006); Efendi, Srivastava and Swanson (2007); Cornett, Marcus and Tehranian (2008).
} 
a large set of numbers describing the profitability of each single activity. ${ }^{3}$ Under this metaphor, our key assumption is that firms are able to package activities in the firm as they wish, but not to hide them. All activities must be reported, and they cannot be made more or less visible to investors. As a result, the average reported profitability must coincide with the true aggregate profitability of the firm. ${ }^{4}$

If investors were able to read and process the entire report provided by a given firm and if, to continue on the packaging metaphor, they had a common understanding of how the various activities affect the future profitability of the firm, the way in which activities are packaged would make no difference. Investors would obtain a common assessment of each firm's value and that would coincide with the true value of the firm. Yet, there is ample evidence that investors tend to end up with different beliefs about firms' values even when exposed to the same reports, as illustrated for example by the abnormal trading volumes frequently observed around earning announcements. Scholars have also noted that disagreement tends to be more pronounced when the financial reports are more complex. ${ }^{5}$ Such evidence suggests that either investors base their estimates (at least partly) on different pieces of information in the reports or that they interpret this information differently.

Motivated by this observation, we assume, in our model, that investors independently of each other pay attention to $K$ activities/signals, taken at random, from the report of each firm, and they assess the value of the firm based on the average profitability observed on these sampled activities. One interpretation is that investors tend to overextrapolate the value of firms from the possibly small sample of activities they pay attention to. An alternative interpretation is that investors hold heterogeneous beliefs as to which activities are more representative of each firm's value (and our model assumes a special form of heterogeneity). The key implication is that investors may end up with different valuations of each firm,

\footnotetext{
${ }^{3}$ We wish to capture the idea that, in practice, firms have a lot of discretion in the way they report their performance to investors. Even relatively simple reports, like earnings announcements, are typically supplemented by a large set of information such as balance sheets, cash flows, and earnings disaggregated at various levels (say by products or geographic regions). The amount of additional information provided, as well as its format, is largely discretionary (Chen, DeFond and Park (2002); Francis, Schipper and Vincent (2002)).

${ }^{4}$ This does not require that the regulator knows the profitability of the firm ex-ante but rather that he may be able to observe it ex-post.

${ }^{5}$ See e.g. Beaver (1968); Kandel and Pearson (1995); Hong and Stein (2007), and in particular Bailey, Li, Mao and Zhong (2003); Sarkar and Schwartz (2009); Hope, Thomas and Winterbotham (2009) on the role of complex information. See also Morgan (2002); Flannery, Kwan and Nimalendran (2004) for studies in which firms' opacity and investors' disagreement appear so closely interrelated that the latter is used as a proxy for the former.
} 
which is what motivates trade in our setting.

Because the average of what each firm reports is correct, and draws from the reports of firms are made independently across investors, it follows that investors' estimates are on average correct. One might have thought that as a result no significant price distortions should arise. Yet, this intuition is incorrect. Prices need not reflect the average belief across all investors, and, as our analysis will reveal, for well-chosen distributions, prices can exceed such average beliefs.

We develop our insights in a simple setting in which investors are risk-neutral and can only trade one stock, either short sell or buy. Hence, they trade the stock for which they expect the highest gains from trade (that is, the highest difference between their perceived values of the firms and the corresponding prices).

We first show that, in a monopolistic setting, the firm can make sure that the valuation of the marginal investor exceeds the average valuation (typically using some skewed distribution of returns). We show that the mispricing obtained in our simple setup carries over to more general specifications, as long as investors' demand is not linear in the perceived gains from trade. Moreover, we show that mispricing can persist even if investors become more sophisticated in the sense that the sample size on which they base their estimates grows very large.

We then turn to our main question of interest, which concerns the effect of increasing the number of firms competing for investors' trades. The key observation is that, in an oligopolistic setting, firms can exploit an additional source of manipulation. Since each investor only trades a subset of firms, the price of firm $j$ reflects the valuations of those who trade firm $j$ (as opposed to the valuations of all investors). A well-chosen reporting strategy can distort prices by making investors who trade firm $j$ be those who are on average more optimistic about $j$.

To illustrate this most simply, we consider the symmetric equilibrium which induces the highest stock price. In this equilibrium, the distribution of beliefs induced by each firm $j$ is positively skewed: a few investors end up with very positive evaluations and many investors end up with moderately negative evaluations about $j$. In this way, when considering which firms to trade, investors tend to perceive higher gains from trading based on good evaluations than on bad evaluations. Since each investor concentrates on trades perceived as the most profitable, the probability of short selling firm $j$ given a negative assessment about $j$ is much smaller than the probability of buying firm $j$ given a positive assessment about $j$. This is what leads to overpricing. Moreover, the effect is all the more pronounced when many firms compete for investors' trades. Fixing firms' reports, the probability of drawing a negative signal from all firms -that is the only case in which 
selling occurs- decreases with the number of firms. In this way, low evaluations are less likely to be incorporated into prices, which implies that overpricing increases with the number of firms in the market. ${ }^{6}$

We perform our main analysis in a stylized model which abstracts from many (relevant) features of real world financial markets. In Section 6, we discuss the robustness of our insights to various modifications of this basic setting. We show that our key results can be derived when investors' demand depends in a smoother way on perceived gains from trade and when investors perform some (heuristic) form of inference from market prices. We also discuss the effects of imposing that firms' reports must include an aggregate summary of their profitability or that reported values cannot exceed an upper bound, or that the sampling process is correlated among investors or that some share of investors would be fully rational or that the fundamental values would be heterogeneous and private information to firms.

We believe our findings have some important implications for the study of manipulation of beliefs and mispricing in financial markets. An important observation that comes out from our analysis is that disagreement may lead to overpricing even when beliefs are on average correct and there is no asymmetry in investors' ability to buy or sell stocks (in particular, short selling is allowed). In our model, such an asymmetry arises endogenously as a result of firms' strategic choice of financial report, which makes negative aspects of the report less likely to affect prices than good aspects. ${ }^{7}$ Observe that firms are not simply interested in creating disagreement, as the shape of disagreement matters. In particular, as explained above, positively skewed distributions of beliefs are key to sustain overpricing in our oligopolistic setting. We also notice that the skewness increases with the number of firms competing to attract traders.

From a policy perspective, we think these insights can be informative for the above mentioned debate on firms' transparency and investors' sophistication. In this respect, we highlight that a form of transparency whereby no activity can be hidden from the reports does not ensure market efficiency. In our setting, transparency is not about providing more information. In a sense, our firms provide more information than what is needed to assess their value. In a similar vein, increasing investors' sophistication is not only about increasing how much infor-

\footnotetext{
${ }^{6}$ This argument only shows why market clearing may occur at a higher price as we increase the number of firms. A more complete description of why no firm can unilaterally deviate and get a higher price, as well as a discussion of other possible equilibria, is left to Section 5 .

${ }^{7}$ This is to be contrasted with the insight in Harrison and Kreps (1978) that the most optimistic trader fixes the price, which crucially hinges on the impossibility of short-selling.
} 
mation they can process. ${ }^{8}$ While we show that this may reduce mispricing, it need not eliminate it, as long as investors underestimate the strategic content of firms' reports. Institutions that specialize in deciphering firms' reports, such as rating agencies, appear useful in this respect. At the same time, however, rating agencies may increase the degree of correlation in investors' evaluations and, as we observe in Section 6, that may exacerbate mispricing.

The rest of the paper is organized as follows. In the next subsection, we review our approach in relation to the existing literature. In particular, we report evidence which motivates our interest in overextrapolation in financial markets and we discuss alternative models that have dealt with similar motivations. In Section 2 we present our baseline model. In Section 3 we analyze a market with a single firm in the simplest sophistication scenario. In Section 4 we study the effect of sophistication. In Section 5 we study the effect of increasing the number of firms. Section 6 offers a discussion of the robustness of our results and of the role of bounded rationality in our setting. Section 7 concludes.

\subsection{Related literature}

\subsubsection{Overextrapolation}

A key aspect of our model is that investors end up with different beliefs even if exposed to the same reports, and they are willing to trade based on these beliefs. Having different interpretations of the same information can be related to models of limited attention. Chahrour (2014) considers a rational inattention model in which when too much information is transmitted by the central bank, investors end up having different beliefs because they randomly sample different aspects of the information. Our model is however different in spirit from Chahrour (2014) and more generally from most of the rational inattention literature started by Sims (2003). First, it does not view the information acquisition as being optimally determined based on some prior knowledge of the problem. ${ }^{9}$ Second, and importantly, our investors are not rational in the sense of making the correct inference from the signals they get and from market prices. Compared to the rational

\footnotetext{
${ }^{8}$ Information overload has been widely discussed across social disciplines, including accounting (see Eppler and Mengis (2004) for a survey). Its policy implications are heatedly debated. We refer to Paredes (2003) for an interesting discussion of information overload from a regulatory perspective.

${ }^{9}$ See e.g. Bordalo, Gennaioli and Shleifer (2012), Köszegi and Szeidl (2013), Gabaix (2013) and Pavan (2014) for alternative behavioral modelling touching on targeted attention, and Di Maggio and Pagano (2013) for a model of disclosure in which investors have different abilities to process complex information.
} 
inattention literature, our investors have no knowledge about the distribution of information in the economy and they rely exclusively on the limited set of signals they pay attention to.

The tendency to overextrapolate from small samples that our modelling of investors' heuristics assumes is well documented in the psychological literature. It may reflect for example what Tversky and Kahneman (1971) called the "law of small numbers" whereby "people regard a sample randomly drawn from a population as highly representative, that is, similar to the population in all essential characteristics." Evidence of overextrapolation appears also explicitly in the context of financial markets. Several surveys show how investors' expectations are strongly influenced by a small sample of past returns (see e.g. Shiller (2000); Dominitz and Manski (2011); Greenwood and Shleifer (2014)); similar evidence appears also in studies of actual investment decisions. ${ }^{10}$

The specific formalization of the heuristic followed by our investors builds on the sampling heuristic first studied by Osborne and Rubinstein (1998) in a gametheoretic context and then applied in IO settings by Spiegler (2006a) and Spiegler $(2006 b) .{ }^{11}$ Our model follows the spirit of Spiegler also in the questions that are being addressed (effect of sophistication, effect of competition), but our application is different, leading to different formulations of the game and different conclusions.

Alternative models of investors' overextrapolation have been considered in the context of financial markets. De Long, Shleifer, Summers and Waldmann (1990b) study in a multi-period setting whether arbitrageurs have a stabilizing role in the presence of extrapolative investors, while Barberis, Shleifer and Vishny (1998), Rabin (2002) and Rabin and Vayanos (2010) focus on how extrapolative investors react to news. None of these papers studies the issue of strategic financial reporting, which is the main focus of our paper.

\footnotetext{
${ }^{10}$ Benartzi (2001) show that employees' investment in company stock depends heavily on past returns (and that is not correlated to future returns); Greenwood and Nagel (2009) document that inexperienced fund managers contributed to the Internet bubbles by chasing trends; Baquero and Verbeek (2008) show that money tend to flow from poorly performing hedge funds to funds with good past performance (and this does not improve future returns). A common feature in these examples is that agents choose their investment strategy by overextrapolating from the limited amount of data they observe.

${ }^{11}$ See also Rubinstein and Spiegler (2008), who consider a speculative market in which investors randomly sample one price in the history of posted prices and buy if the current price is below the sampled price.
} 


\subsubsection{Other related work}

A few recent papers analyze the formation of stock prices in competitive equilibria with non-omniscient agents (in Gul, Pesendorfer and Strzalecki (2011), agents can only distinguish a limited number of contingencies; in Eyster and Piccione (2013) and Steiner and Stewart (2012), agents rely on coarse reasoning to analyze the dynamics of the market). An essential distinctive feature of our study is the focus on how investors' beliefs may be manipulated, which has no counterpart in these papers. ${ }^{12}$ In addition, a growing literature studies financial markets in which investors hold heterogeneous beliefs (see Scheinkman and Xiong (2004) and Hong and Stein (2007) for reviews). Our model seems to be among the first to investigate how firms may use financial reports as a way to influence investors' disagreement.

There is also a large literature that studies how much information can be transmitted from an informed sender to an uninformed decision-maker when the latter is assumed to be perfectly rational. The literature provides various possible modelling of the disclosure choice of senders. The sender may choose its strategy after having observed the state (as in models of cheap talk à la Crawford and Sobel (1982)). Or he can commit to a disclosure policy ex ante (as in Kamenica and Gentzkow (2011), Rayo and Segal (2010) or Jehiel (2011)). Or else he can be forbidden to lie other than by omission (as in Grossman (1981), Milgrom (1981), Shin (2003)). Some scholars have adapted such models to the study of financial markets. For example, Stein (1989) considers a model in which rational investors correctly anticipate that managers distort earnings and so markets are efficient. Our approach shares with that literature -in particular the Bayesian persuasion literature- the desire to endogenize the degree of transparency/opaqueness chosen here by firms regarding their communication strategy. ${ }^{13}$ As already mentioned, however, we do not assume that investors perform rational inferences from the signals they receive and instead base their decisions on simpler heuristics. In line with the evidence reported above, this seems a useful complementary approach for studying complex situations in which investors may lack a complete Bayesian

\footnotetext{
${ }^{12}$ In Hong, Scheinkman and Xiong (2008), subjective beliefs are derived in a setting in which analysts provide biased recommendations and investors are heterogeneous in their ability to draw the correct inference from these recommendations. In their setting, however, the objective for analysts is to appear of "good type" as opposed to manipulate investors' beliefs about firms' values.

${ }^{13}$ In particular, Kamenica and Gentzkow (2011) identify conditions under which the sender can benefit from communication, typically by using noisy signals where noise is adjusted so as to induce the best choice of action at minimal cost.
} 
representation of the environment they are facing. We further elaborate on the relation with fully rational models in Section 6 .

Finally, the strategies of firms in financial reporting are analyzed in a large literature in accounting (see e.g. Verrecchia (2001) for a survey, and Hirshleifer and Teoh (2003) for a model in which investors have limited attention). This literature, however, generally abstracts from the role of improved investors' sophistication and of market competition on firms' reporting strategies, which is the main focus of our analysis.

\section{Model}

Consider a stock market consisting of $F$ firms $j=1, \ldots F$, each having overall profitability $\varphi$, which we interpret as firms' fundamental value. There is a unitary mass of investors trading on the stock market. Investors are unaware of the fundamental values of the firms. They assess the profitability of the various firms by sampling the profitability of some (randomly drawn) activities in the various firms and extrapolating from the sample. Each investor can only trade one unit of one stock (either buy or short sell), and he trades the stock for which he perceives the highest gain from trade. The prices of the various stocks are determined through market clearing conditions.

Firms are assumed to know the procedure followed by investors, and they seek to maximize the price of their stocks. Each firm chooses a financial reporting strategy that consists in a set of signals representing the returns of the various activities in the firm. Firms are free to package (or even fabricate) activities as they wish and we refer to this strategic choice as their financial report. The mean of these signals is constrained to coincide with the fundamental value $\varphi$, which holds because of regulatory constraints (all activities must appear somewhere). Moreover, firms are constrained to not report too low returns, and we normalize the lower bound to zero.

There is complete information among firms, and we consider the Nash equilibria of the financial reporting game played by the firms. In particular, our analysis will focus on whether the prices of the stocks differ from the fundamental values, and how the sophistication of investors (see below for a measure of sophistication) and/or the degree of competitiveness (as measured by the number $F$ of firms) affect the result.

Formally, the packaging of firm $j$ activities in the financial report is described 
as a distribution $\sigma^{j}$, whose support $X^{j}$ satisfies

$$
X^{j} \subset \mathbb{R}_{+},
$$

and whose mean $\bar{x}^{j}$ satisfies

$$
\bar{x}^{j}=\varphi,
$$

for each $j=1, \ldots, F{ }^{14}$ We denote by $\Sigma$ the set of signal distributions satisfying conditions (1)-(2), and we allow firms to choose any distribution in $\Sigma$. In the sequel, we refer to (2) as the aggregation condition.

Investors do not know the fundamental values of the firms, and they employ a simple heuristic procedure in order to assess them. For each firm, they consider $K$ independent random draws from the signal distribution of the firm, and they interpret the average of these $K$ signals as the fundamental value of the firm.

Hence, if investor $i$ observes signals $x_{i, 1}^{j}, x_{i, 2}^{j}, \ldots, x_{i, K}^{j}$ from firm $j$, his assessment of the value of firm $j$ is

$$
\hat{x}_{i}^{j}=\frac{1}{K} \sum_{n=1}^{K} x_{i, n}^{j} .
$$

We also assume that the draws are independent across investors and firms. Such a heuristic can easily be interpreted along one of the general principles outlined in Kahneman (2011): "All there is (for investor $i$ ) to assess firm $j$ 's value is what investor $i$ sees of firm $j$," and we implicitly assume here that investor $i$ only sees $x_{i, n}^{j}, n=1, \ldots, K$ of firm $j$, from which $\hat{x}_{i}^{j}$ is obviously a focal assessment. ${ }^{15}$

Based on his assessments of the values of firms, each investor trades one unit of one stock and he can either buy or short sell it. Hence, investor $i$ is willing to trade one unit of stock $r$ if stock $r$ is perceived to offer the highest gains from trade. That is, if

$$
r \in \arg \max _{j}\left|p^{j}-\hat{x}_{i}^{j}\right|
$$

where $p^{j}$ denotes the price of stock $j$. Investor $i$ buys stock $r$ if $p^{r} \leq \hat{x}_{i}^{r}$ and he short sells stock $r$ if $p^{r} \geq \hat{x}_{i}^{r}$. Note that $\arg \max _{j}\left|p^{j}-\hat{x}_{i}^{j}\right|$ may sometimes consist of several stocks $r$, in which case investor $i$ is indifferent between several options. In

\footnotetext{
${ }^{14}$ It should be noted that, while for convenience we describe a firm's report as a distribution of signals, there is nothing stochastic in such report. Randomness only arises as a result of investors' sampling procedure.

${ }^{15}$ That $\hat{x}_{i}^{j}$ is focal can possibly be related to a form of coarse reasoning. If one has to form a guess as to what the mean of a distribution is on the basis of $K$ independent draws from the distribution, then without further information (meaning by averaging over all possible distributions) the empirical mean would be the right guess. Such a line of reasoning can be modeled using Jehiel (2005)'s analogy-based expectation equilibrium.
} 
case of indifference, a tie-breaking rule (to be determined endogenously) specifies the probability assigned to the various possible trades.

Formally, let $\sigma=\left\{\sigma^{j}\right\}, p=\left\{p^{j}\right\}$ and $\hat{x}_{i}=\left\{\hat{x}_{i}^{j}\right\}, j=1, . ., F$. A tie-breaking rule $\omega$ specifies for each $\left(p, \hat{x}_{i}\right)$ a probability $\omega_{r}\left(p, \hat{x}_{i}\right)$ of demanding (resp. shortselling) one stock of firm $r$ if $p^{r} \leq \hat{x}_{i}^{r}$ (resp. $p^{r} \geq \hat{x}_{i}^{r}$ ) with the requirement that $\omega_{r}\left(p, \hat{x}_{i}\right) \neq 0$ only if $r \in \arg \max _{j}\left|p^{j}-\hat{x}_{i}^{j}\right|$.

Any given $\sigma$ induces a distribution over $\hat{x}_{i}$. The law of large number guarantees that, for finite economies and as the number of investors gets large, the distribution of $\hat{x}_{i}$ across individuals $i$ would get close to $\sigma$ (for any topology if $\sigma$ is a distribution of finitely many signals and for appropriately chosen topologies otherwise). Working directly with a continuum of investors, we assume that the distribution of $\hat{x}_{i}$ across individuals $i$ coincide with $\sigma .{ }^{16}$ Formally, given $\hat{x}_{i}, p$, $\omega, i$ 's demand of stock $j$ is $d_{i}^{j}\left(\hat{x}_{i}, p, \omega\right)=\omega_{j}\left(p, \hat{x}_{i}\right)$ if $p^{j} \leq \hat{x}_{i}^{j}$ and 0 if $p^{j}>\hat{x}_{i}^{j}$. Similarly, $i$ ' supply of stock $j$ is $s_{i}^{j}\left(\hat{x}_{i}, p, \omega\right)=\omega_{j}\left(p, \hat{x}_{i}\right)$ if $p^{j} \geq \hat{x}_{i}^{j}$ and 0 if $p^{j}<\hat{x}_{i}^{j}$. Thanks to our identification assumption, the aggregate demand and supply can then be computed according to:

$$
\begin{aligned}
D^{j}(\sigma, p, \omega) & =\int d_{i}^{j}\left(\hat{x}_{i}, p, \omega\right) d \hat{x}_{i}, \\
S^{j}(\sigma, p, \omega) & =\int s_{i}^{j}\left(\hat{x}_{i}, p, \omega\right) d \hat{x}_{i} .
\end{aligned}
$$

As far as firms are concerned, we assume that they are completely rational and that they know the procedure employed by investors (in particular, they know $K)$. Given that firm $j$ seeks to maximize $p^{j}$, this leads to the following definition of equilibrium (in which $\sigma^{-j}$ and $p^{-j}$ are the distributions and prices for all firms except $j$ ):

Definition 1 (Equilibrium) The profile $(\sigma, p, \omega)$ is an equilibrium if: for each $j$, $\sigma^{j} \in \Sigma$,

a) $D(\sigma, p, \omega)=S(\sigma, p, \omega)$, and

b) There is no distribution $\tilde{\sigma}^{j} \in \Sigma$, prices $\tilde{p}^{j}, \tilde{p}^{-j}$, and tie-breaking rule $\tilde{\omega}$ such that $D\left(\tilde{\sigma}^{j}, \sigma^{-j}, \tilde{p}^{j}, \tilde{p}^{-j}, \tilde{\omega}\right)=S\left(\tilde{\sigma}^{j}, \sigma^{-j}, \tilde{p}^{j}, \tilde{p}^{-j}, \tilde{\omega}\right)$ and $\tilde{p}^{j}>p^{j}$.

Condition (a) requires that the markets clear. Condition (b) requires that there should be no profitable deviation for any firm $j$, where a profitable deviation

\footnotetext{
${ }^{16}$ There are well known technical issues associated with the use of the law of large numbers with a continuum of independent variables (see for example, Judd (1985)). We abstract from these issues here, even though we conjecture our results hold approximately in finite economies as the number of investors gets large (for prices nearby those shown in the various propositions with a probability close to 1 ).
} 
$\tilde{\sigma}^{j}$ of firm $j$ means that for the profile of distributions $\left(\tilde{\sigma}^{j}, \sigma^{-j}\right)$, there exists a tie-breaking rule $\tilde{\omega}$ and prices $\tilde{p}^{j}, \tilde{p}^{-j}$ that clear the markets which are such that firm $j$ achieves a strictly higher price $\tilde{p}^{j}>p^{j} \cdot{ }^{17}$

In the following analysis, we will prove the existence of an equilibrium (in a constructive manner). Discrete distributions with a finite number of signals will play an important role. We will denote by $\sigma=\left\{x_{1}, \mu_{1} ; x_{2}, \mu_{2} ; ..\right\}$ the distribution in which $x_{1}$ is reported with probability $\mu_{1}, x_{2}$ is reported with probability $\mu_{2}$, and so on.

\section{Monopoly}

We first focus on a monopolistic firm facing investors who just consider one activity in the financial report. That is, we set $F=1$ and $K=1$.

As there is only one firm, the market clearing price corresponds to the median belief about the firm's value. At this price, half of the investors want to buy and half of them want to sell. Since each investor only trades one unit, the market clears. Moreover, given that investors only consider one signal, such a median belief corresponds to the median of the firm's distribution. Hence, the monopoly's problem is to choose a distribution with the maximal median that satisfies the constraints (1) and (2) that signals should be non-negative and that the mean of the distribution should coincide with the fundamental $\varphi$.

Such maximization is achieved with a two-signal distribution that puts weight on 0 and $h$ and such that the median is just $h$ (requiring that the weight on $h$ is just above that on 0 ). To see this, observe that any signal strictly above the median is a waste for the firm as reducing such a signal to the median while increasing all signals slightly so as to meet the aggregation condition (2) would be profitable. Similarly, any signal strictly in between 0 and the median is a waste, as lowering such signals to 0 while increasing all signals slightly so as to respect (2) would be profitable. Consider then $\sigma=\{0,1-\mu ; h, \mu\}$ with $\mu \geq 1 / 2$. The aggregation condition (2) implies that $\mu h=\varphi$, and thus the maximum price that can be achieved by the monopolist is $2 \varphi$. The following Proposition collects these

\footnotetext{
${ }^{17}$ There are alternative possible definitions of profitable deviations (based on other expectations about the ensuing market clearing prices). Note however that any equilibrium as defined here would a fortiori be an equilibrium under the alternative specifications of profitable deviations. None of our results depends on this specific choice.
} 
observations. ${ }^{18}$

Proposition 1 Suppose $F=1$ and $K=1$. The firm chooses the distribution $\sigma_{M}=\{0,1 / 2 ; 2 \varphi, 1 / 2\}$. The price is $p_{M}=2 \varphi$.

\section{Monopoly and Sophistication}

We now turn to a setting in which investors are more sophisticated in the sense of considering larger samples. More precisely, we consider a monopolist and we assume that investors sample several $(K>1)$ signals in order to evaluate the fundamental value of the firm. Our question of interest is whether the price gets close to the fundamental if we let $K$ be sufficiently large.

Based on the law of large number, one might have expected that, for $K$ large enough, investors would end up with (approximately) correct assessments of the fundamental value, and thus the market clearing price would have to be close to $\varphi$. Such an intuition would be true if the financial reporting strategy of the firm were set independently of $K$. But, this is not the relevant consideration here, given that the firm can adjust its financial reporting strategy to the number of draws made by investors (since we assume that firms know $K$ before they choose their reporting strategy). Thus, the distribution chosen by the firm will typically change with $K$, and the law of large number need not apply.

In fact, we show that to the extent that $K$ is finite, no matter how large, the firm can guarantee a price bounded away from the fundamental by a suitable choice of reporting strategy. By the previous argument, such reporting strategy must depend on $K$. Specifically, consider the following two-signal distribution:

$$
\sigma_{K}=\left\{0,(1 / 2)^{1 / K} ; h(K), 1-(1 / 2)^{1 / K}\right\}
$$

and the price $p_{K}=h(K) / K$, with $h(K)=\varphi /\left[1-(1 / 2)^{1 / K}\right]$ so that the mean of the distribution is $\varphi$.

An investor who gets $K$ draws from the distribution and samples $z$ times the signal $h(K)$ is willing to buy if the price does not exceeds $z h(K) / K$. As the price equals $h(K) / K$, only those who sample $K$ times signal 0 are willing to sell, which is a proportion $\left[(1 / 2)^{1 / K}\right]^{K}=1 / 2$ of investors. That is, at this price half of investors sell and half of the investors buy, so the market clears.

\footnotetext{
${ }^{18}$ The tie-breaking rule is the one favoring demand over supply in case of indifference. Accordingly, when the price is equal to their evaluation, investors are assumed to be buying one unit of stock.
} 
So given $K$, the monopolist can achieve a price of its stock no smaller than

$$
p_{K}=\frac{\varphi}{K\left[1-(1 / 2)^{1 / K}\right]}
$$

Simple algebra reveals that $p_{K}$ is decreasing with $K$ and that $p_{K}$ converges to $\varphi / \ln 2$, which is strictly bigger than $\varphi$, as $K$ grows arbitrarily large. Hence, we have established:

Proposition 2 Suppose $F=1$. Irrespective of $K$, the firm can attain a price no smaller than $\varphi / \ln 2$, which is strictly larger than $\varphi$.

As described in (4), the distribution used to establish Proposition 2 requires that there is no upper bound on the signals that can be sent by the firm $(h(K)=$ $\varphi /\left[1-(1 / 2)^{1 / K}\right]$ goes to infinity as $K$ goes to infinity). If there were an upper bound (as considered in Section 6), the variance of the distribution would have to be bounded, and the firm would not be able to obtain a price of its stock much away from the fundamental when $K$ is large.

\section{Oligopoly}

We now turn to our main questions of interest: How does an increase in the number of firms competing in the stock market affect the mispricing of stocks and can the mispricing if any persist as the number of firms grows large?

It is not a priori clear in which way increasing the number $F$ of firms may drive the mispricing. Inducing a higher market clearing price would require attracting more demand and so tilt the financial reporting distribution toward higher signals. Yet, since the mean of the distribution has to coincide with the fundamental, that would have to be counter-balanced by having more weight on low signals, which would trigger more supply. This makes it hard to identify how the most relevant deviations would look like and so what effect competition may have on stock prices. We divide our investigation into various subsections. Our main result appears in Proposition 5.

\subsection{A non-transparency result}

A first observation is that no matter how many firms are competing on the stock market, it cannot be an equilibrium that (all) firms choose a transparent financial reporting interpreted as a reporting distribution concentrated on the fundamental 
value. Indeed, if all firms choose $\sigma=\{\varphi, 1\}$, then obviously the market clearing price for all stocks is $p=\varphi$ and no firm would be perceived as offering any gain from trade. But, suppose that firm $j$ chooses the distribution displayed in the monopoly case; that is, $\sigma^{j}=\{0,1 / 2 ; 2 \varphi, 1 / 2\}$ when $K=1$. Then trading other stocks at price $\varphi$ would be viewed as offering no gains from trade, and as a result one can assume that all trades take place on stock $j$. As shown in Section 3, firm $j$ can obtain a price of its stock as high as $2 \varphi$, thereby showing that the deviation is profitable. This observation carries over to any specification of $K$ (by Proposition 2), thereby allowing us to derive:

Proposition 3 Irrespective of $F$ and $K$, there is no equilibrium in which firms choose as their reporting strategy a distribution concentrated on the fundamental value.

A second observation is that, irrespective of the strategy used by others, a firm can always guarantee that the price of its stock is at least the fundamental value. Indeed if firm $j$ chooses $\sigma^{j}=\{\varphi, 1\}$ then $p^{j}=\varphi$ is necessarily a market clearing price for $j$ (and there is no other possible market clearing price for $j$ if some of the stocks $j$ are to be traded). This establishes the following Proposition:

Proposition 4 In all equilibria, the price of stocks is no smaller than the fundamental value.

\subsection{The highest price equilibrium}

Characterizing all equilibria is somewhat difficult because it requires getting into comparative statics properties of the Walrasian equilibria of the stock market as induced by the various possible choices of reporting strategies of the firms (which in turn affect in a complex way the demand and supply of the various stocks through the sampling heuristic). ${ }^{19}$

To keep the analysis tractable, we consider the case in which investors only consider one activity in the financial report, i.e. $K=1$. Moreover, we restrict our attention to symmetric equilibria. That is, we require that in equilibrium firms choose the same distribution of signals, the prices of the various stocks are the same, and the tie-breaking rule is anonymous. ${ }^{20}$ Among symmetric equilibria, we

\footnotetext{
${ }^{19}$ The theory of general equilibrium has essentially produced existence and efficiency results but very few instances in which Walrasian prices can be explicitly derived from the demand and supply structure. For our purpose, it is the latter that is required though.

${ }^{20}$ That is, if a mass $\mu$ of investors ends up with the same assessment about a set of $N$ firms, each of these firms receives a fraction $\mu / N$ of the trades.
} 
focus on the equilibrium that induces the highest prices of stocks. There are two ways to think of such a focus: 1) It highlights how much the prices can be far from the fundamental. 2) It is a natural benchmark equilibrium if we have in mind that the firms in the stock market can coordinate on the equilibrium they like best. We will also in the next subsection discuss other (symmetric) equilibria, thereby showing the range of prices that can be sustained in equilibrium.

In order to characterize the highest price symmetric equilibrium, we proceed in several steps. First, we characterize among the symmetric distributions of signals and anonymous tie-breaking rules the ones that induce the largest common clearing price of stocks. Then, we show that such a symmetric distribution of signals and anonymous tie-breaking rule together with the corresponding profile of prices constitutes an equilibrium, thereby leading to a characterization of the highest price symmetric equilibrium.

\subsubsection{Market clearing}

Consider a strategy profile $\{\sigma, p, \omega\}$, such that each firm chooses the same distribution $\sigma=\left\{x_{1}, \mu_{1} ; x_{2}, \mu_{2} ; \ldots\right\}, p$ denotes the common market clearing price and $\omega$ is an anonymous tie-breaking rule. We first note that $\sigma$ may induce the highest price in this class only if it satisfies the following property: There must be no signal $x>0$ which is in the support of $\sigma$ and such that signal $\tilde{x}=2 p-x$ is not in the support of $\sigma$. That is, all positive signals in the support of $\sigma$ need to be paired around the price. To see this, suppose by contradiction that $\sigma$ assigns mass $\mu_{x}>0$ to an unpaired signal $x$ (i.e., there is no mass on $2 p-x$ ). Suppose also for the sake of the argument that $x>p$. Then one could obtain the same price by moving $x$ to the lower adjacent signal $\hat{x}$ in the support of $\sigma$ (or to $p$ if there is no signal between $x$ and $p$ ). The average of the distribution would be reduced by $\mu_{x}(x-\hat{x})$. This would then allow us to increase all signals and so the price by the same amount, thereby showing that the distribution did not induce the highest price. In what follows, we say that $\widehat{\sigma} \in \hat{\Sigma}$ if $\widehat{\sigma} \in \Sigma$ (as defined by conditions (1) and (2)) and all positive signals in $\widehat{\sigma}$ are paired around some $p$ interpreted as the price.

The second step in our argument is to observe that to achieve the largest price, the distribution $\hat{\sigma} \in \hat{\Sigma}$ should assign positive weight to at most three signals. To see this, suppose that $\hat{\sigma}$ assigns positive weight to $n$ signals and $n>3$. Then one

can define another distribution $\sigma \in \hat{\Sigma}$ which involves at most $n-1$ signals and that induces a price $\tilde{p} \geq p$ (assuming again an anonymous tie-breaking rule and 
that $\sigma^{j}=\tilde{\sigma}$ for all $j$ ). The idea is to remove the two signals closest to the price and move their mass either to the price (if the weight of the higher of the two signals is no smaller than the weight of the smaller one) or to the adjacent signals further away from the price (if the weight of the smaller signal is bigger than the weight of the higher signal), and then increase all signals and the price upward so as to accommodate the aggregation condition.

Iterating the argument, one gets a distribution with at most three signals, $0, p, 2 p$. Then, one can move equal mass from $p$ to 0 and $2 p$ or vice-versa without changing the market clearing price. Thus, we end up with a two-signal distribution which takes one of the following forms: $\sigma_{a}=\left\{0,1-\mu_{a} ; 2 p_{a}, \mu_{a}\right\}$ or $\sigma_{b}=\left\{0,1-\mu_{b} ; p_{b}, \mu_{b}\right\}$. Consider $\sigma_{a}$. Investors are indifferent between trading stock $j$ and stock $r$ whenever they sample signal $2 p_{a}$ from firm $j$ and signal 0 from firm $r$. The highest aggregate demand is obtained by letting investors buy $j$ whenever indifferent between buying $j$ and selling another stock. In that case, the aggregate supply includes only the mass of those traders who sample signal 0 from all firms, which has probability $\left(1-\mu_{a}\right)^{F}$. Hence, market clearing requires $\left(1-\mu_{a}\right)^{F} \leq 1 / 2$. If $\left(1-\mu_{a}\right)^{F}<1 / 2$, one can decrease slightly $\mu_{a}$ and increase all signals by $\varepsilon$ and obtain a price which is $\varepsilon$ higher. Hence, among distributions $\sigma_{a}$, the price is maximized by setting $\mu_{a}=\mu^{*}$ where

$$
\mu^{*}=1-(1 / 2)^{1 / F}
$$

The highest market clearing price from distributions $\sigma_{a}$ is thus obtained with

$$
\sigma^{*}=\left\{0,1-\mu^{*} ; \varphi / \mu^{*}, \mu^{*}\right\}
$$

and the resulting market clearing price is

$$
p^{*}=\frac{\varphi}{2 \mu^{*}}
$$

With simple algebra, one can show that no distribution in $\sigma_{b}$ can achieve a price which is higher than $p^{*}$. This in turn leads to the next Lemma, whose detailed proof appears in the Appendix.

Lemma 1 Assume that for some $\widehat{\sigma} \in \Sigma, \sigma^{j}=\widehat{\sigma}$ for all $j$, and consider an anonymous tie-breaking rule. The resulting market clearing price $\widehat{p}$ is no larger than $p^{*}$, as defined in (7). Moreover, $p^{*}$ is obtained with the distribution $\sigma^{*}$, as defined in (5) and (6). 


\subsubsection{Deviations}

We now show that when $\sigma^{j}=\sigma^{*}, p^{j}=p^{*}$ for all $j$, no firm can profitably deviate, so indeed the distribution $\sigma^{*}$ and the price is $p^{*}$ together with the anonymous tie-breaking rule that favors demand over supply in case of indifference define an equilibrium.

Lemma 2 There is a symmetric equilibrium in which firms choose the distribution $\sigma^{*}$ and the price is $p^{*}$, as defined respectively in (6) and (7).

To get a sense of why Lemma 2 holds true, consider $\left(\sigma^{*}, p^{*}, \omega^{*}\right)$ where $\sigma^{*}$ and $p^{*}$ are defined by (6) and (7) and $\omega^{*}$ is the tie-breaking rule favoring demand over supply in case of indifference. Assume by contradiction that there is a deviation by one firm, say firm $j$ that would lead to a market clearing price $p^{j}>p^{*}$ for firm $j$ and a market clearing price $p^{\prime}$ for the non-deviating firms (in the Appendix, we allow for the case in which the non-deviating firms have different market clearing prices) and let $h=2 p^{*}\left(=\varphi / \mu^{*}\right)$.

We first note that $p^{\prime} \leq h / 2$ as $p^{\prime}>h / 2$ would imply excess supply for the non-deviating firm and thus make market clearing impossible (remember that $\left(1-\mu^{*}\right)^{F-1}>1 / 2$ whenever $\left.\left(1-\mu^{*}\right)^{F}=1 / 2\right)$.

We next observe that the total weight on signals of firm $j$ above $p^{j}+p^{\prime}$ should be no smaller than $\mu^{*}$ as otherwise there would be excess aggregate supply over the stocks of all firms (remember again that $\left(1-\mu^{*}\right)^{F}=1 / 2$ ).

If $p^{\prime}=h / 2$ as before firm $j$ 's deviation, the aggregation condition (2) would not hold given the observation just made that the weight of firm $j$ ' signals above $p^{*}+p^{\prime}$ should be no smaller than $\mu^{*}$, and $p^{j}>h / 2=p^{\prime}$.

One may then wonder whether having a smaller $p^{\prime}<h / 2$ could help alleviating the constraint (2). To see that this cannot be the case, observe that the total weight of firm $j$ signals strictly below $p^{j}-p^{\prime}$ cannot exceed some threshold $x$ as otherwise there would be excess supply for firm $j$ (investors sell stock $j$ whenever such a signal is drawn with signals 0 from the non-deviating firms). Besides, such a threshold $x$ gets arbitrarily small as $F$ grows large.

Given the above observations, the mean of the distribution of signals of firm $j$ is no smaller than $\mu^{*}\left(p^{j}+p^{\prime}\right)+\left(1-\mu^{*}-x\right)\left(p^{j}-p^{\prime}\right)$, but since $x$ is small this expression decreases with $p^{\prime}$. As for $p^{\prime}=h / 2$ it is strictly larger than $\varphi$, the aggregation condition (2) cannot be satisfied for any $p^{\prime} \leq h / 2$.

We conclude from the above that there can be no deviation of firm $j$ that could possibly induce a market clearing price $p^{j}$ strictly above $p^{*}=h / 2$, thereby establishing Lemma 2. 


\subsubsection{Main result}

Combining Lemmas 1 and 2, we have our main result:

Proposition 5 Suppose $K=1$ and $F>1$. The maximal price achieved in a symmetric equilibrium is $p^{*}(F)=\frac{\varphi}{2\left[1-\left(\frac{1}{2}\right)^{1 / F}\right]}$. This price increases in $F$.

The reason why the price of stocks $p^{*}$ increases with $F$ is as follows. The price of a given firm $j$ must reflect the median (here also the average) of the valuations of those who trade firm $j$. This however differs from the average valuation across all investors, as not all investors trade all firms. That is what opens the possibility of distorting prices when many firms compete in the market. More precisely, in the equilibrium of Proposition 5, investors sell stock $j$ only when they sample $F$ low signals (from all firms). ${ }^{21}$ For a given frequency of high signal, the more firms, the lower the chance that the signals drawn from all firms are low. In this way, bad evaluations are less likely to be reflected in market prices when the number of firms increases, and as a result the price $p^{*}$ increases with $F$.

A question arises as to how the market clearing price in the competitive case compares with the monopoly price (see Proposition 1) for various F. Simple calculations reveal that the price in the duopoly case is smaller than in the monopoly case, but the price for any other market structure configuration $(F>2)$ is larger than in the monopoly case. ${ }^{22}$

It should also be noted that in our setting the total number of signals that investors pay attention to increases with the number of firms (since investors consider one signal from each firm irrespective of $F$ ). In this way, we highlight the effect of competition among firms over investors' trades rather than over investors' attention. ${ }^{23}$ One may ask how our construction would be affected if investors could process at most $S$ signals say, and so they would sample one signal from at most $S$ firms. Proposition 5 would hold by substituting $F$ with $\min \{F, S\}$. When $F>S$, our result would then be interpreted as showing that mispricing increases when investors are allowed to pay attention to more firms.

\footnotetext{
${ }^{21}$ Notice that this tie-breaking rule is not imposed ex ante but it is determined in equilibrium. In fact, given the distribution $\sigma^{*}$ chosen by the firms, this is the only tie-breaking rule which allows to reach an equilibrium as defined in Definition 1.

${ }^{22}$ These considerations imply that if a monopolistic firm could split its activity into several companies with different stocks, it would benefit from it given the heuristic of the investors.

${ }^{23}$ Hirshleifer, Lim and Teoh (2009) provide evidence consistent with the idea that information is less likely to be incorporated in market prices when many signals compete for investors' attention.
} 
Finally, we conjecture that our insight that more competition may drive the price further away from the fundamental would continue to hold had we considered arbitrary $K>1$. While the analysis would become quite cumbersome, we conjecture that the above arguments can be replicated by considering the distribution $\sigma=\{0,1-\mu ; h, \mu\}$, where $\mu$ is defined by $(1-\mu)^{F K}=1 / 2$ and the price $p^{*}=\varphi / 2 \mu K^{24}$

\subsection{Other Equilibria}

We now highlight that, apart from the highest price symmetric equilibrium described above, other (symmetric) equilibria may arise. To illustrate this, we exhibit a symmetric equilibrium that induces a market clearing price as low as the fundamental (which combined with Propositions 4 and 5 allows us to show the range of market clearing prices that can be sustained in symmetric equilibria). More precisely, we have:

Proposition 6 Suppose $K=1$. For every $F>1$, there is a symmetric equilibrium with market clearing prices $p=\varphi$. The common distribution of signals has support $(0,2 \varphi)$. It is centered around $\varphi$, and it is such that the probability of sampling $F-1$ signals within distance $z$ from $\varphi$ is linear in $z$. When $F=2$, it is the uniform distribution on $(0,2 \varphi)$.

To get some intuition for Proposition 6, consider the duopoly case $F=2$. If firm 1 chooses a uniform distribution of signals between 0 and $2 \varphi$, it is not hard to see that irrespective of the choice of distribution of firm 2 , the market clearing price for firm 1 must be $p_{1}=\varphi$. Indeed at this price, and given the symmetry of the distribution of firm 1 around $\varphi$, there is as much demand as there is supply for firm 1 (whatever the choice of distribution of firm 2). More important for our purpose though is the observation that when firm 1 chooses such a distribution, the market clearing price of firm 2 cannot be larger than $\varphi$. If the support of the distribution of firm 2 coincides with $(0,2 \varphi)$, one can show that the market clearing price of the two firms has to be $\varphi$. This is because 1) any signal $s_{2}>p_{2}$ generates a demand for firm 2 proportional to $s_{2}-p_{2}$ (that corresponds to the probability that $s_{1}$ satisfies $\left|s_{1}-p_{1}\right|<s_{2}-p_{2}$ conditional on $\left.s_{2}\right), 2$ ) any signal

\footnotetext{
${ }^{24}$ Notice that, for $K$ large and $F$ small, this price may not exceed the fundamental. The comparative statics exercise should then be conducted for $F$ sufficiently large.
} 
$s_{2}<p_{2}$ generates a supply for firm 2 proportional to $p_{2}-s_{2}$, and 3 )

$$
\int_{\varphi}^{2 \varphi}\left(s_{2}-\varphi\right) f\left(s_{2}\right) d s_{2}=\int_{0}^{\varphi}\left(\varphi-s_{2}\right) f\left(s_{2}\right) d s_{2},
$$

for all densities $f(\cdot)$ with support $(0,2 \varphi)$ satisfying the aggregation condition (2). Moreover, any positive measure of signal above $2 \varphi$ would lead to a strictly lower price for firm 2. This in turn establishes Proposition 6 for the duopoly case and the argument can be generalized for an arbitrary number of firms (see the Appendix).

Two further comments about the equilibrium displayed in Proposition 6 are worth mentioning. First, as $F$ increases, the corresponding distribution of signals becomes more concentrated around $\varphi$ (so for this equilibrium, more competition eventually induces financial reports that get close to reporting just their fundamental value). Second, the equilibrium shown in Proposition 6 suffers from the following fragility. While the equilibrium requires that firms choose a distribution with continuous density, an obvious alternative (and simpler) best-response would be for the firms to report just their fundamental value. Yet, if firms were to choose such a financial reporting strategy, this would not be an equilibrium (see Proposition 3).

\section{Discussion}

In this Section, we discuss the role of our main assumptions. We break the discussion into several subsections. We first consider our main assumptions on investors: bounded rationality and trading limits. We then consider a number of other extensions. We consider the case in which the financial report distribution must be bounded from above, then the case in which it must contain a summary statistic regarding the aggregate profitability. We also discuss the possibility that fundamental values be asymmetric and private information to firms. Finally, we discuss the effect of correlation in investors' draws and of the presence of some fully rational investors in our setting.

\subsection{Bounded rationality}

We first discuss in somewhat general terms the role of bounded rationality in our setting, we then analyze the robustness of our results to modifications of our specific modeling of bounded rationality. 


\subsubsection{Why bounded rationality}

A key question is which of our insights could be derived in a setting with fully rational investors. Specifically, what if investors in our setting could draw correct inferences from the signals they receive and the levels of prices (as in Grossman and Stiglitz (1980), say)?

In order to make the problem interesting, our basic setup should be somewhat modified. First, we should assume that fundamental values are stochastic and private information to firms. Otherwise, any investor if rational would assess the value of the firms as $\varphi$ irrespective of the signals received. Second, we should introduce some reasons to trade which are not purely speculative. Otherwise, as long as investors hold a common prior about the distributions of fundamental values, they would not be willing to trade in equilibrium (Milgrom and Stokey (1982)) even if firms were to engage in strategic information disclosure about the realization of their fundamental values. Suppose one introduces noise traders (as in Grossman and Stiglitz (1980) or in De Long, Shleifer, Summers and Waldmann $(1990 a)$ ) who would trade not based on informational inferences. In a setting in which the mass of such investors would be stochastic, the inference that rational traders could make from the price would be imperfect. As Albagli, Hellwig and Tsyvinski (2012) show, in a setting with trading constraints, prices may depart from fundamentals even when informed investors employ standard Bayesian reasoning.

Bounded rationality in our setting should not be viewed just as a way to generate mispricing (which, as just mentioned, may occur also with Bayesian investors). Rather, it is a way to provide a precise structure on the behavior of non-fully rational traders. Apart from being of interest in itself (as discussed above, we think this is a behaviorally plausible heuristic), such an explicit foundation is required so as to define how firms can influence beliefs, and then address our main question of how manipulations may depend on the degree of sophistication of investors and of market competition.

The sign of the mispricing due to noise traders (as in De Long et al. (1990a) or in Albagli et al. (2012)) may be difficult to predict in traditional financial market models in which non-noise traders are assumed to be fully rational. Indeed, the mispricing may go in either direction depending on how information and the mass of noise traders are distributed (see Albagli et al. (2012)). This is different from our approach in which bounded rationality induces predictable deviations from fundamentals. Moreover, as the behaviors of noise traders are usually set 
exogenously in those models, it is not clear how firms would be able to influence it. Finally, in the above mentioned literature, the set of signals from which (rational) traders gather information is typically set exogenously. Our key interest is instead in deriving these signals from the reporting strategies chosen by the various firms.

This makes our focus quite distinct from the literature on financial markets with heterogeneous beliefs and noise traders and we think it offers a different perspective. The perspectives should be viewed as complementary.

\subsubsection{Alternative heuristics}

One way in which our investors could be made more sophisticated is by enriching the set of elements they consider when assessing firms' values. For example, investors could consider that the price itself is indicative of the fundamental value. Alternatively, investors could base their estimates not only on the part of the financial report they pay attention to but also on the market sentiment (De Long et al. $(1990 a))$. Finally, investors could take into account that their estimate is noisy and adjust their investment decision accordingly.

There are several possible ways to incorporate such ideas into the heuristic of investors; we review some of them. In the main model, investor $i$ made an estimate of the fundamental value of firm $j$ based on the average sample signal $\hat{x}_{i}^{j}$ from $j$. Suppose instead that investor $i$ assesses the fundamental value of firm $j$ according to

$$
v_{i}^{j}=\lambda_{i} p^{j}+\left(1-\lambda_{i}\right) \hat{x}_{i}^{j},
$$

where $\lambda_{i} \in[0,1)$ reflects the subjective weight attached by investor $i$ to the informativeness of the price relative to the informativeness of the private signal $\hat{x}_{i}^{j}$. Trading $j$ would be assessed to give gains of $\left|p^{j}-v_{i}^{j}\right|=\left(1-\lambda_{i}\right)\left|p^{j}-\hat{x}_{i}^{j}\right|$ and thus, our previous analysis would apply equally to this new specification. In a richer model, the weight $\lambda_{i}$ could be derived endogenously (and it could a priori depend on $j$ as well). The (specific) reduced-form approach in (8) is meant to illustrate that introducing some coarse inference from the price need not change the logic developed above.

The idea of "market sentiment" can be modeled using the average belief of the various investors about the profitability of the firm. As already noted, in our setting, the average belief about firm $j$ corresponds to its fundamental value. Thus, an investor $i$ receiving an average sample $\hat{x}_{i}^{j}$ from $j$ would assess firm $j$ according to

$$
v_{i}^{j}=\tau \varphi+(1-\tau) \hat{x}_{i}^{j},
$$


where $\tau \in[0,1)$ represents the weight given to the market sentiment. The gains from trade attached to asset $j$ would be perceived as $\left|p^{j}-\tau \varphi-(1-\tau) \hat{x}_{i}^{j}\right|$, and the main messages of our previous analysis would remain qualitatively the same. As $K$ grows large in the monopoly case, the price would be bounded by $\tau \varphi+(1-\tau) \varphi / \ln 2$, which exceeds $\varphi$. In the oligopoly case with $K=1$, as $F$ grows large, the maximal price sustainable in equilibrium would be $\tau \varphi+(1-\tau) p^{*}$, where $p^{*}$ denotes the price characterized in Section 5.

Finally, we could incorporate the idea that investors would take into account that their estimate of the fundamental value is noisy. For example, when investors draw several signals $K>1$, instead of simply considering the mean of the signal and reason as if it were the fundamental value, investors could also consider the empirical variance in the sample and reason as if the fundamental value was a random variable normally distributed with mean and variance coinciding with the corresponding empirical values in the sample. With risk neutral investors (as we assumed) this would have no consequence. With risk-averse investors, it is not clear a priori in which way our main analysis would change given that both buying and short selling would be perceived as risky. A more systematic investigation of such heuristics should be the subject of future work.

\subsection{Trading constraints}

Assuming that investors can only trade one unit of one stock is, of course, special. More generally, trade orders may be smooth functions of the perceived gains from trade, and investors may trade several firms.

Consider the monopoly case $F=1$ with a sample size $K=1$. Letting $\hat{x}_{i}$ and $p$ denote, respectively, the perceived value and price of the stock, we denote by $f\left(\left|\hat{x}_{i}-p\right|\right)$ the demand (resp. supply) for the stock if $\hat{x}_{i}-p>0$ (resp. $\hat{x}_{i}-p<0$ ), and we assume that $f(\cdot)$ is a smooth (in particular, continuous) function, thereby implying that $f(0)=0$.

Given that $f(0)=0$, it is clear that it would not be possible to achieve a price equal to the highest possible signal as in Proposition 1 (since at that price there would be no demand). It is also clear that if $f(\cdot)$ is linear everywhere the price would correspond to the average of investors' beliefs, which would be equal to the fundamental value. As we now show, however, the firm can achieve a price strictly above the fundamental whenever $f(\cdot)$ is non-linear (at least somewhere between 0 and $\varphi$ ). Suppose for example that $f(\cdot)$ is strictly concave and the firm chooses the distribution $\{\varphi-\varepsilon-\delta, 1 / 2-\nu ; \varphi+\varepsilon, 1 / 2+\nu\}$, where $\varepsilon, \delta$ and $\nu$ are 
positive. At $p=\varphi$, the demand would be $(1 / 2+\nu) f(\varepsilon)$ while the supply would be $(1 / 2-\nu) f(\varepsilon+\delta)$. By concavity of $f(\cdot)$, the demand would exceed the supply and so market clearing would require $p>\varphi$. More generally, we have:

Proposition 7 Suppose that $K=F=1$ and $f(\cdot)$ is strictly concave or strictly convex in a neighborhood $W \subseteq[0, \varphi]$. The firm can achieve a price $p>\varphi$.

The above proposition establishes that the kind of distortions exhibited in the monopoly case would continue to hold whenever the demand is not linear in the perceived gains from trade. We believe that there are many practical reasons why such non-linearities could arise. For example, if investors face wealth and short selling constraints, they may not be able to trade as much as they wish of a given firm. Alternatively, if they face trading costs, they may not trade when perceived gains are too small. More generally, one can think of portfolio choice models in which investors consider increasing their exposure to a given stock by trading off perceived expected benefit against perceived increase in risk. Outside the case of normally distributed risk and CARA preferences, smooth non-linear demand/supply functions may naturally arise.

Consider next the oligopoly case with $F>1$. Observe first that in the equilibrium considered in Proposition 5, both buyers and sellers perceive the same gain from trade (that is, $p^{*}$ ). Hence, the market clearing condition is not affected by the specific form of the function $f(\cdot)$. Moreover, as we now show, no firm can profitably deviate by choosing alternative reporting strategies provided that $f(\cdot)$ is sufficiently concave.

Proposition 8 Suppose that $K=1, F>1$ and that $f(x)=x^{z}$, with $z<1 / 2$. There is a symmetric equilibrium in which firms choose the distribution $\sigma^{*}$ and the price is $p^{*}$, as defined respectively in (6) and ( 7$)$.

To get an intuition for the result, suppose a firm deviates and offers some very high signals. As demand is now strictly increasing in gains from trade, this would tend to push the price of the deviating firm up. At the same time, other signals must be decreased in order to meet the aggregation condition (2), and this tends to push the price down. If $f(\cdot)$ does not increase sufficiently fast with gains from trade, the second effect dominates, and the proposed deviation is not profitable. The proof extends the argument to arbitrary deviations. We conclude that the insight that mispricing may increase with the number of firms, and in particular 
the equilibrium construction developed in Proposition 5, does not depend on the extreme form of demand considered in the main analysis. ${ }^{25}$

Similarly, the effect of competition appears robust to the possibility that investors (for reasons which we have not introduced) may trade several firms. Suppose for example that investors would trade the $M$ most profitable firms, where $M<F$. Following the logic of Proposition 5, investors may be induced to sell only if they end up with fewer than $M$ good evaluations, and this is less likely to occur as $F$ gets large. Hence, to the extent that an investor cannot trade all stocks, prices would be less affected by bad evaluations as many firms compete in the market, and we conjecture that our main conclusion that competition may magnify mispricing would continue to hold.

\subsection{Further extensions}

\subsubsection{Upper bound on firms' reports}

In our baseline model, firms were free to report signals with arbitrarily large values. One may question how our results would be affected if we were to impose an upper bound on firms' distribution. As already mentioned, the logic developed in Proposition 2 would not hold in this case, and a monopolistic firm would not be able to obtain a price much larger than $\varphi$ when the sample size $K$ gets arbitrarily large. By contrast, and perhaps more interestingly, the result that competition need not eliminate and may even increase mispricing would be preserved.

To see this, assume that the support of the distribution used by firm $j$ must be in the range $[0, H]$. If $H \geq \varphi / \mu^{*}(F)$, where $\mu^{*}(F)$ is defined in (5), then the upper bound does not bind and the previous analysis applies. Consider then $H<\varphi / \mu^{*}(F)$ and assume that $H \geq \tilde{H}$, where

$$
\tilde{H}=\max \left\{\frac{\varphi}{1-2 \mu^{*}(F)}, 2 \varphi\right\}
$$

As we show in the next Proposition, the highest symmetric equilibrium price in this case is achieved with the distribution $\sigma_{H}=\left\{l, 1-\mu^{*}(F) ; H, \mu^{*}(F)\right\}$ in which the tie-breaking rule is anonymous and most favorable to demand and $\mu^{*}$ is defined

\footnotetext{
${ }^{25}$ The analysis of the case in which demand would be of the form $f(x)=x^{z}$ with $z>\frac{1}{2}$ is left for future research.
} 
as in (5) by the market clearing condition $\left(1-\mu^{*}\right)^{F}=1 / 2 .{ }^{26}$ This price is

$$
p_{H}(F)=\frac{H+l(F)}{2},
$$

where due to the aggregation condition (2)

$$
l(F)=\frac{\varphi-\mu^{*}(F) H}{1-\mu^{*}(F)} .
$$

By the same logic as that explained in Section $5, \mu^{*}$ decreases in $F$, which allows to increase $l$ and so $p_{H}$. In the limit as $F$ gets arbitrarily large, $l$ gets close to $\varphi$ and $p_{H}$ converges to $(\varphi+H) / 2$. That is, as in our previous analysis, the maximal equilibrium price increases in the number of competing firms, but now the maximum price never goes beyond $(\varphi+H) / 2$. We collect these observations in the following Proposition.

Proposition 9 Suppose that signals must be in the range $[0, H]$ and $H \geq \tilde{H}$, as defined in (10). The maximal price achieved in a symmetric equilibrium increases in $F$, and converges to $(\varphi+H) / 2$ as $F$ gets arbitrarily large.

This result sheds some light on a policy intervention intended to impose an upper bound on reports (whereby for example reporting too high profitability in some dimensions would lead to investigations). First, such a bound may be difficult to define based on the behavior of similar firms in the market. The equilibrium we describe is symmetric so in a sense no firm appears "too profitable" relative to its competitors. Second, even if such an upper bound were implemented, it would affect the level of mispricing but not the potentially detrimental role of competition in our setting.

\subsubsection{Reporting overall profitability}

One may object to our main modelling assumptions that real world financial reports typically contain a mention of the aggregate profitability of the firm in addition to more disaggregated details that motivated our main model. If investors only paid attention to these aggregate figures, there would be no disagreement

\footnotetext{
${ }^{26}$ As detailed in the proof, the condition $H \geq 2 \varphi$ ensures that $\sigma_{H}$ induces a higher price than a three signals distribution which puts positive mass on signals $0, s$ and $H$ and induces a price $(s+H) / 2$. The condition $H \geq \varphi /\left(1-2 \mu^{*}\right)$ ensures that $\sigma_{H}$ induces a higher price than a two signals distribution which puts positive mass on signals 0 and $h$ and induces a price $h$.
} 
between them. Yet, we believe this view is at odds with the evidence that disagreement between investors tends to increase upon the release of financial reports (see the references in footnote 5). To be in line with this evidence, we propose that while investors would put some weight $\tau$ on the aggregate profitability, they would also put some complementary weight $1-\tau$ on disaggregated profitability data in an attempt to make a better estimate about future profitability of the firms. Since investors may hold different beliefs as to which activities will affect most future profitability, we could consider that investor $i$ assesses the fundamental value of firm $j$ according to $v_{i}^{j}=\tau \varphi+(1-\tau) \hat{x}_{i}^{j}$, where $\hat{x}_{i}^{j}$ is the average profitability among activities considered as most representative of firm $j$ by investor $i$, and $\varphi$ is the aggregate profitability as truthfully reported by firm $j$. That formulation would be equivalent (in reduced form) to that of equation (9).

\subsubsection{Asymmetric and/or stochastic fundamentals}

We have so far assumed that all firms have the same fundamental value $\varphi$, which is deterministic and commonly known among firms. We consider relaxing each of these assumptions. First, note that extending the definitions of the equilibrium to the cases of asymmetric and/or stochastic fundamentals raises no difficulties. Second, we now show that the logic of our analysis extends to these cases.

Clearly, in the monopoly case, nothing would be changed by allowing the fundamental $\varphi$ to be randomly determined. For each realization of $\varphi$, the obtained price would be the one derived above for this value of the fundamental. More challenging though is the analysis of competition when fundamental values may be asymmetric and/or stochastically determined.

Consider first the case of asymmetric (though deterministic) fundamentals. Firm $j$ has fundamental value $\varphi^{j}=\varphi+\varepsilon^{j}$ and assume that $0 \leq \varepsilon^{1} \leq \varepsilon^{2} \leq \ldots \varepsilon^{F}$ (without loss of generality). The following proposition identifies an equilibrium in the same spirit as the one described in Proposition 5, in which $\mu^{*}(F)=1-(1 / 2)^{1 / F}$ and $p^{*}(F)=\varphi / 2 \mu^{*}(F)$ as defined in equations (5) and (7). This equilibrium requires that heterogeneity among firms is not too large. In particular, as detailed below, it requires

$$
\varepsilon^{F} \leq \bar{\varepsilon}(F)
$$

where

$$
\bar{\varepsilon}(F) \equiv\left\{\begin{array}{ccc}
\varphi \sqrt{2} / 2 & \text { for } & F=2 \\
2\left(p^{*}-\varphi\right) & \text { for } & F>2
\end{array}\right.
$$

Proposition 10 Suppose firm $j$ has fundamental value $\varphi^{j}=\varphi+\varepsilon^{j}$, and as- 
sume that $K=1$ and $\varepsilon^{F} \leq \bar{\varepsilon}(F)$. There is an equilibrium in which $\sigma^{j}=$ $\left\{\varepsilon^{j}, 1-\mu^{*}(F) ; 2 p^{*}(F)+\varepsilon^{j}, \mu^{*}(F)\right\}$ and the prices are $p^{j}=p^{*}(F)+\varepsilon^{j}$ for all $j$. These prices increase in $F$.

Given that $\bar{\varepsilon}(F)$ grows arbitrarily large as $F$ increases, if for all $i, \varepsilon^{i}<\tilde{\varepsilon}$ for some constant $\tilde{\varepsilon}$, then for $F$ large enough, it must be that $\varepsilon^{F}<\bar{\varepsilon}(F)$ and thus Proposition 10 applies. Moreover, in this equilibrium, adding more firms has the effect of increasing $p^{*}(F)$ and thus the price of all firms.

Compared to Proposition 5, the main difference is that firm $j$ may consider using signals lower than the lowest signal in the support of the reporting strategy (given that $\varepsilon^{j}>0$ ). The reason for the extra condition (12) can be understood as follows. First, firm $j$ with fundamental value $\varphi^{j}=\varphi+\varepsilon^{j}$ can deviate and choose the distribution $\left\{0,1 / 2 ; 2 \varphi+2 \varepsilon^{j}, 1 / 2\right\}$. At prices $p^{j}=2 \varphi+2 \varepsilon^{j}-p^{*}$ and $p^{r}=p^{*}$ for $r \neq j$, firm $j$ would attract all investors and the market would clear. For this to be unprofitable, it should be that $p^{*}+\varepsilon^{j} \geq 2 \varphi+2 \varepsilon^{j}-p^{*}$, which must hold in particular for the most profitable firm, thereby explaining that $\varepsilon^{F} \leq 2\left(p^{*}(F)-\varphi\right)$ is required. Second, when $F=2$, a deviation of firm $j$ to $\left\{0, \mu^{*} ; p^{j}, 1-\mu^{*}\right\}$ would induce prices $p^{j}=\left(\varphi+\varepsilon^{j}\right) /\left(1-\mu^{*}\right)$ and $p^{r}=\varepsilon^{r}$ for $r \neq j$. For this to be unprofitable, it should be that $p^{*}(F)+\varepsilon^{j} \geq \sqrt{2}\left(\varphi+\varepsilon^{j}\right)$, which for the most profitable firm writes $\varepsilon^{F} \leq \varphi \sqrt{2} / 2$. It turns out that all deviations are taken care of when $\varepsilon^{F}<\bar{\varepsilon}(F)$, as defined above.

It should be noted that in the equilibrium of Proposition 10, the strategy of firm $j$ depends only on her own fundamental value $\varphi^{j}$. This has nice implications for the case in which the fundamental values would be stochastically drawn. Indeed, assume that the fundamental value $\varphi^{j}$ of firm $j, j=1 \ldots F$, is now stochastically drawn from a distribution with support $[\varphi, \varphi+\bar{\varepsilon}(F)]$, and that only firm $j$ knows the realization of $\varphi^{j}$. Define for each firm $j$ receiving the fundamental value $\varphi^{j}$ the strategy $\sigma^{j}\left(\varphi^{j}\right)=\left\{\varepsilon^{j}, 1-\mu^{*}(F) ; 2 p^{*}(F)+\varepsilon^{j}, \mu^{*}(F)\right\}$ where $\varepsilon^{j}=\varphi^{j}-\varphi$, together with the price $p^{j}\left(\varphi^{j}\right)=p^{*}(F)+\varepsilon^{j}$. Because such strategies constitute an ex-post equilibrium (i.e. they remain in equilibrium after the realization of all fundamental values are known), we have:

Proposition 11 The above strategies and prices are part of a Bayes-Nash equilibrium whatever the joint distribution of fundamentals.

Together Propositions 10 and 11 show that our main results regarding the destabilizing effect of competition is robust to the introduction of asymmetries, private information, and randomness in firms' fundamental values. 


\subsubsection{Correlation in investors' draws}

We have so far assumed that the draws from firms' reports are made independently across investors. As discussed in the Introduction, this allows us to generate heterogeneous beliefs and that is what generates trade in our setting. It should be noted that, from a theoretical perspective, such an assumption is a priori the most favorable to market efficiency. Introducing some systematic correlation in investors' draws, e.g. allowing that some signals are known to receive more attention than others, typically weakens the effect of condition (2) and is likely to increase the scope for distortions. Yet, as a complement to our benchmark model with independent draws, it may be interesting to explore the effect of correlation in investors' draws. For example, such a correlation may capture the idea that investors are influenced by financial analysts who often look at the same aspects of the financial reports.

As a step toward this, suppose that each investor pays attention to $N+K$ signals from each firm's report. Among them, signals $x_{1}, . ., x_{N}$ are observed by all investors. The remaining $K$ signals are sampled independently across investors from the rest of the report. Investor $i$ assesses the value of firm $j$ as

$$
v_{i}^{j}=\frac{N}{K+N} \hat{x}^{j}+\frac{K}{K+N} \hat{x}_{i}^{j}
$$

where $\hat{x}^{j}$ denotes the average of signals $x_{1}^{j}, . ., x_{N}^{j}$ and $\hat{x}_{i}^{j}$ denotes the average of the signals sampled from the rest of the report. Since firms can choose reports with an arbitrarily large number of signals, the aggregation condition (2) is not affected by the chosen values $x_{1}, . ., x_{N}$. It follows that if firms know which signals investors commonly pay attention to, they would report arbitrarily large profitability in these signals and they could induce arbitrarily large evaluations. If instead reports are bounded from above by $H$ as in Section 6.3.1, firms would set $x_{s}=H$ for $s=1, . ., N$ and choose the remaining signals so as to maximize the market clearing price subject to condition (2). The problem is similar to the one analyzed in the baseline model and the previous insights can be applied. If $F=K=1$ and $H \geq 2 \varphi$, it follows from the analysis of Section 3 that the firm can induce a price

$$
p_{N}=\frac{N}{1+N} H+\frac{1}{1+N} 2 \varphi
$$

Similarly, the analysis of Section 4, 5 and 6.3 .1 can be used to define the corresponding price as one increases $K$ or $F$. Hence, the effects identified in our main model remain qualitatively unchanged. 
Suppose instead that firms do not know the set of signals which is commonly observed by all investors. That creates some randomness on the market clearing price. But, the previous analysis extends by noting that firms expect a valuation $\varphi$ from the $N$ signals commonly observed, so that the expected price now is a weighted average between $\varphi$ and the price as derived in the main analysis. Of course, the randomness on the price would be exacerbated if one considered a market with a small number of investors (or with a small number of analysts who mediate between firms and investors). A more complete analysis of such extensions should be the subject of future research.

\subsubsection{Introducing rational investors}

We now consider the effect of introducing some fully rational investors. Suppose investors are either fully rational $(K=\infty)$ with probability $\alpha$ or they follow the $K$ - sampling procedure (with $K<\infty$ ) with probability $1-\alpha$. In line with our baseline model, we assume that investors whether fully rational or boundedly rational can trade only one stock, and that the fundamental values of all firms are the same.

Given that the fundamental value of each firm is deterministic, rational investors know it with certainty. Given that the price is typically above the fundamental value, rational investors would all go for short selling. Our equilibrium constructions of Sections 3, 4, 5 should then be modified by adding a share $\alpha$ to the aggregate supply. In Section 4, market clearing would require $(1-\mu)^{K}(1-\alpha)+\alpha=1 / 2$. In Section 5 , it would require $(1-\mu)^{F}(1-\alpha)+\alpha=1 / 2$. One can replicate the same analysis as above with the modified market clearing conditions. It is not difficult to show that, provided $\alpha$ is not too large, our previous insights carry over.

Notice that the same logic would apply if we assumed that assets were in positive net supply. The level of prices would mechanically decrease (given that investors have a limited buying capacity), but the comparative statics would remain unchanged.

\section{Conclusion}

This paper has considered a stylized financial market in which firms strategically frame their financial reports so as to influence investors' beliefs and induce higher stock prices. We have illustrated how the introduction of less sophisticated, ex- 
trapolative investors in such a setting could alter dramatically the analysis of market efficiency. We have shown that a form of investor protection requesting that overall there should be no lie in the financial reporting need not restore market efficiency. Moreover, capital market competition has been shown to be ineffective in ensuring that prices are close to fundamentals.

Our model is obviously stylized and open to several extensions. In particular, it would be interesting to explore more generally the incentives to manipulate beliefs as a function of which investors -along the distribution of beliefs- are key to determine the market price. Another interesting extension would be to add a time component to the belief formation given that some forms of accounting manipulation occur over time (see e.g. Fudenberg and Tirole (1995)). Finally, future research may also explore the empirical implications of the model. Our results suggest that the complexity of information provided by firms should be positively correlated to investors' disagreement and to trading prices. In particular, according to Proposition 5, positively skewed distribution of beliefs should be associated with overpricing, and this effect should be stronger in settings in which many firms compete for investors' trades. To our knowledge, this link remains to be explored empirically.

From a broader perspective, even though our paper emphasizes the extrapolative nature of investors' heuristics, the results reported here can be viewed as illustrative of a more general theme. It has long been understood, since Harrison and Kreps (1978), that speculative trade can arise if investors have heterogeneous (subjective) beliefs, say about the profitability of the various firms. What our approach suggests is that firms may try to influence the formation of subjective beliefs, here through their release of financial reports. We have seen how, within our framework, such manipulation could lead to overpricing in the stock market. We believe such a theme of subjective belief manipulation should be the subject of active research in the future, as it seems relevant to explain a number of dysfunctionings in financial markets.

\section{References}

Albagli, E., Hellwig, C. and Tsyvinski, A. (2012), 'A theory of asset prices based on heterogeneous information', Mimeo. 
Bailey, W., Li, H., Mao, C. X. and Zhong, R. (2003), 'Regulation fair disclosure and earnings information: Market, analyst, and corporate responses', Journal of Finance 58(6), 2487-2514.

Baquero, G. and Verbeek, M. (2008), 'Do sophisticated investors believe in the law of small numbers?', Mimeo.

Barberis, N., Shleifer, A. and Vishny, R. (1998), 'A model of investor sentiment', Journal of Financial Economics 49(3), 307-343.

Beaver, W. H. (1968), 'The information content of annual earnings announcements', Journal of Accounting Research 6, pp. 67-92.

Benartzi, S. (2001), 'Excessive extrapolation and the allocation of 401(k) accounts to company stock', Journal of Finance 56(5), 1747-1764.

Bergstresser, D. and Philippon, T. (2006), 'Ceo incentives and earnings management', Journal of Financial Economics 80(3), 511-529.

Bordalo, P., Gennaioli, N. and Shleifer, A. (2012), 'Salience theory of choice under risk', Quarterly Journal of Economics 127(3), 1243-1285.

Burns, N. and Kedia, S. (2006), 'The impact of performance-based compensation on misreporting', Journal of Financial Economics 79(1), 35-67.

Chahrour, R. (2014), 'Public Communication and Information Acquisition', American Economic Journal: Macroeconomics 6(3), 73-101.

Chen, S., DeFond, M. L. and Park, C. W. (2002), 'Voluntary disclosure of balance sheet information in quarterly earnings announcements', Journal of Accounting and Economics 33(2), 229 - 251.

Cornett, M. M., Marcus, A. J. and Tehranian, H. (2008), 'Corporate governance and pay-for-performance: The impact of earnings management', Journal of Financial Economics 87(2), 357-373.

Crawford, V. P. and Sobel, J. (1982), 'Strategic information transmission', Econometrica 50(6), 1431-51.

De Long, J. B., Shleifer, A., Summers, L. H. and Waldmann, R. J. (1990a), 'Noise trader risk in financial markets', Journal of Political Economy 98(4), 703-38. 
De Long, J. B., Shleifer, A., Summers, L. H. and Waldmann, R. J. (1990b), 'Positive feedback investment strategies and destabilizing rational speculation', Journal of Finance 45(2), 379-395.

Di Maggio, M. and Pagano, M. (2013), 'Financial disclosure with limited processing capacity', Mimeo.

Dominitz, J. and Manski, C. F. (2011), 'Measuring and interpreting expectations of equity returns', Journal of Applied Econometrics 26(3), 352-370.

Efendi, J., Srivastava, A. and Swanson, E. P. (2007), 'Why do corporate managers misstate financial statements? the role of option compensation and other factors', Journal of Financial Economics 85(3), 667-708.

Eppler, M. J. and Mengis, J. (2004), 'The concept of information overload: A review of literature from organization science, accounting, marketing, mis, and related disciplines', The information society 20(5), 325-344.

Eyster, E. and Piccione, M. (2013), 'An approach to asset pricing under incomplete and diverse perceptions', Econometrica 81(4), 1483-1506.

Flannery, M. J., Kwan, S. H. and Nimalendran, M. (2004), 'Market evidence on the opaqueness of banking firms' assets', Journal of Financial Economics 71(3), 419-460.

Francis, J., Schipper, K. and Vincent, L. (2002), 'Expanded disclosures and the increased usefulness of earnings announcements', Accounting Review 77(3), pp. $515-546$.

Fudenberg, D. and Tirole, J. (1995), 'A theory of income and dividend smoothing based on incumbency rents', Journal of Political Economy 103(1), 75-93.

Gabaix, X. (2013), 'A sparsity-based model of bounded rationality, with application to basic consumer and equilibrium theory', Quarterly Journal of Economics - forthcoming.

Greenwood, R. and Nagel, S. (2009), 'Inexperienced investors and bubbles', Journal of Financial Economics 93(2), 239-258.

Greenwood, R. and Shleifer, A. (2014), 'Expectations of returns and expected returns', Review of Financial Studies 27(3), 714-746. 
Grossman, S. J. (1981), 'The informational role of warranties and private disclosure about product quality', Journal of Law and Economics 24(3), pp. 461-483.

Grossman, S. J. and Stiglitz, J. E. (1980), 'On the impossibility of informationally efficient markets', American Economic Review 70(3), 393-408.

Gul, F., Pesendorfer, W. and Strzalecki, T. (2011), 'Behavioral competitive equilibrium and extreme prices', Mimeo.

Harrison, J. M. and Kreps, D. M. (1978), 'Speculative investor behavior in a stock market with heterogeneous expectations', Quarterly Journal of Economics 92(2), 323-36.

Hirshleifer, D., Lim, S. S. and Teoh, S. H. (2009), 'Driven to distraction: Extraneous events and underreaction to earnings news', Journal of Finance 64(5), 2289-2325.

Hirshleifer, D. and Teoh, S. H. (2003), 'Limited attention, information disclosure, and financial reporting', Journal of Accounting and Economics 36(1-3), 337386.

Hong, H., Scheinkman, J. and Xiong, W. (2008), 'Advisors and asset prices: A model of the origins of bubbles', Journal of Financial Economics 89(2), 268287.

Hong, H. and Stein, J. C. (2007), 'Disagreement and the stock market', Journal of Economic Perspectives 21(2), 109-128.

Hope, O.-K., Thomas, W. B. and Winterbotham, G. (2009), 'Geographic earnings disclosure and trading volume', Journal of Accounting and Public Policy 28(3), 167-188.

Jehiel, P. (2005), 'Analogy-based expectation equilibrium', Journal of Economic Theory 123(2), 81-104.

Jehiel, P. (2011), 'On transparency in organizations', Review of Economic Studies - forthcoming.

Judd, K. L. (1985), 'The law of large numbers with a continuum of iid random variables', Journal of Economic Theory 35(1), 19-25.

Kahneman, D. (2011), Thinking, Fast and Slow, Farrar, Straus and Giroux. 
Kamenica, E. and Gentzkow, M. (2011), 'Bayesian persuasion', American Economic Review 101(6), 2590-2615.

Kandel, E. and Pearson, N. D. (1995), 'Differential interpretation of public signals and trade in speculative markets', Journal of Political Economy 103(4), 83172 .

Kőszegi, B. and Szeidl, A. (2013), 'A model of focusing in economic choice', Quarterly Journal of Economics 128(1), 53-104.

Levitt, A. (1998), 'The numbers game', Remarks delivered at the NYU Center for Law and Business, September 28.

Milgrom, P. (1981), 'Good news and bad news: Representation theorems and applications', Bell Journal of Economics 12(2), pp. 380-391.

Milgrom, P. and Stokey, N. (1982), 'Information, trade and common knowledge', Journal of Economic Theory 26(1), 17-27.

Morgan, D. P. (2002), 'Rating banks: Risk and uncertainty in an opaque industry', American Economic Review 92(4), 874-888.

Osborne, M. J. and Rubinstein, A. (1998), 'Games with procedurally rational players', American Economic Review 88(4), 834-47.

Paredes, T. A. (2003), 'Blinded by the light: Information overload and its consequences for securities regulation', Wash. ULQ 81, 417.

Pavan, A. (2014), 'Attention, coordination, and bounded recall', Mimeo.

Rabin, M. (2002), 'Inference by believers in the law of small numbers', Quarterly Journal of Economics 117(3), 775-816.

Rabin, M. and Vayanos, D. (2010), 'The gambler's and hot-hand fallacies: Theory and applications', Review of Economic Studies 77(2), 730-778.

Rayo, L. and Segal, I. (2010), 'Optimal information disclosure', Journal of Political Economy 118(5), $949-987$.

Rubinstein, A. and Spiegler, R. (2008), 'Money pumps in the market', Journal of the European Economic Association 6(1), 237-253.

Sarkar, A. and Schwartz, R. A. (2009), 'Market sidedness: Insights into motives for trade initiation', Journal of Finance 64(1), 375-423. 
Scheinkman, J. A. and Xiong, W. (2004), Heterogeneous beliefs, speculation and trading in financial markets, in R. A. Carmona, E. Çinlar, I. Ekeland, E. Jouini, J. A. Scheinkman and N. Touzi, eds, 'Paris-Princeton Lectures on Mathematical Finance 2003', Springer Berlin / Heidelberg, pp. 223-233.

Shiller, R. J. (2000), 'Measuring bubble expectations and investor confidence', Journal of Psychology and Financial Markets 1(1), 49-60.

Shin, H. S. (2003), 'Disclosures and asset returns', Econometrica 71(1), pp. 105133.

Sims, C. A. (2003), 'Implications of rational inattention', Journal of Monetary Economics 50(3), 665-690.

Spiegler, R. (2006a), 'Competition over agents with boundedly rational expectations', Theoretical Economics 1(2), 207-231.

Spiegler, R. (2006b), 'The market for quacks', Review of Economic Studies 73(4), 1113-1131.

Stein, J. C. (1989), 'Efficient capital markets, inefficient firms: A model of myopic corporate behavior', Quarterly Journal of Economics 104(4), 655-669.

Steiner, J. and Stewart, C. (2012), 'Price distortions in high-frequency markets', Mimeo.

Tversky, A. and Kahneman, D. (1971), 'Belief in the law of small numbers', Psychological Bulletin 76(2), $105-110$.

Verrecchia, R. E. (2001), 'Essays on disclosure', Journal of Accounting and Economics 32(1-3), 97-180.

\section{Appendix}

\section{Proof of Proposition 1}

As shown in the text, the price $p_{M}=2 \varphi$ clears the market when the firm sends the distribution $\sigma_{M}=\{0,1 / 2 ; 2 \varphi, 1 / 2\}$. We now show that no distribution induces a higher price. Suppose that the firm sends the distribution $\sigma=$ $\left\{x_{0}, \mu_{0} ; x_{1}, \mu_{1} ; x_{2}, \mu_{2} ; . . ; x_{N}, \mu_{N}\right\}$ with $0=x_{0}<x_{1}<x_{2}<\ldots<x_{N}$ and $\mu_{n} \geq 0$ for $n=0, \ldots, N$. (We consider a discrete distribution for simplicity of notation, the argument is the same if we consider continuous distributions.) Market clearing 
requires that the price is the median of the distribution. If there are several medians (because of the discreteness of the distribution), then considering the largest median is enough to characterize the largest market clearing price. Thus, we let $p=x_{N}$ if $\mu_{N} \geq 1 / 2 ; p=x_{N-1}$ if $\mu_{N}<1 / 2$ and $\mu_{N}+\mu_{N-1} \geq 1 / 2$; and more generally for $n \in[1, N-1]$

$$
p=x_{n}, \text { if } \sum_{w=0}^{N-n-1} \mu_{N-w}<1 / 2 \text { and } \sum_{w=0}^{N-n} \mu_{N-w} \geq 1 / 2 .
$$

Maximizing $x_{n}$ while satisfying the above constraints and the constraint in (2) requires setting $\mu_{w}=0$ for all $w \in[1, n-1]$. Moreover, by setting $x_{w}=x_{n}$ for all $w \in[n+1, N], x_{n}$ can be increased, and so $p$ can be increased, while still satisfying condition (2). Hence, we are left with a distribution $\sigma=\left\{0,1-\mu ; x_{n}, \mu\right\}$ with $\mu \geq 1 / 2$. Condition (2) requires $x_{n} \leq \varphi / \mu$. As we need $\mu \geq 1 / 2$ to have $p>0$, it follows that $x_{n} \leq 2 \varphi$. Thus, no alternative distribution can induce a price higher than $p_{M}$. Q. E. D.

\section{Proof of Lemma 1}

Part 1. We first show that the distribution $\sigma$ cannot induce the highest price if there is a signal $x>0$ which is in the support of $\sigma$ (that is, to which the distribution $\sigma$ assigns positive mass) and such that signal $\tilde{x}=2 p-x$ is not in the support of $\sigma$. Consider the equilibrium profile $\{\sigma, p, \omega\}$, where $p$ is the market clearing price and $\omega$ is an anonymous tie-breaking rule, and suppose by contradiction that $\sigma$ assigns mass $\mu_{x}>0$ to signal $x$ and no mass to signal $\tilde{x}$. Suppose first that $x>p$ and there is a signal $\hat{x} \in[p, x)$ such that $\sigma$ assigns mass $\mu_{\hat{x}}>0$ to $\hat{x}$ and no mass to any other signal between $x$ and $\hat{x}$. Consider the alternative distribution $\tilde{\sigma}$ in which the mass $\mu_{x}$ is moved to signal $\hat{x}$. Under the original distribution $\sigma$, the demand for each firm can be written as

$$
\begin{aligned}
D=\mu_{x} \sum_{y=0}^{F-1}\left(\begin{array}{c}
F-1 \\
y
\end{array}\right) \frac{1}{F-y}\left(\mu_{x}\right)^{F-1-y}\left(\mu_{\hat{x}}+\mu_{z}\right)^{y}+ \\
\mu_{\hat{x}} \sum_{y=0}^{F-1}\left(\begin{array}{c}
F-1 \\
y
\end{array}\right) \frac{1}{F-y}\left(\mu_{\hat{x}}\right)^{F-1-y}\left(\mu_{z}\right)^{y}+W,
\end{aligned}
$$

where $\mu_{z}$ denotes the total mass of signals that are at a distance to the price smaller than $\hat{x}$ is, $\mu_{z}=\sum_{\left\{n \text { s.t. } 2 p-\hat{x}<x_{n}<\hat{x}\right\}} \mu_{n}$, and $W$ is unaffected by the proposed change in the distribution. The demand for each firm under the new distribution $\tilde{\sigma}$ can 
be written as

$$
\tilde{D}=\left(\mu_{x}+\mu_{\hat{x}}\right) \sum_{y=0}^{F-1}\left(\begin{array}{c}
F-1 \\
y
\end{array}\right) \frac{1}{F-y}\left(\mu_{x}+\mu_{\hat{x}}\right)^{F-1-y}\left(\mu_{z}\right)^{y}+W .
$$

Notice that the supply of each firm is unaffected by the proposed change in the distribution since signal $\tilde{x}=2 p-x$ is not part of $\sigma$. Hence, given that $D=\tilde{D}$, the same market clearing price $p$ can be attained with the new distribution $\tilde{\sigma}$. At the same time, the distribution $\tilde{\sigma}$ has a lower mean than $\sigma$, the difference being $\mu_{x}(x-\hat{x})$. This allows to increase all signals in $\tilde{\sigma}$ and so the price by the same amount, thereby showing that the distribution $\sigma$ did not induce the highest price.

If there is no signal between $x$ and $p$ the same argument applies by moving the mass $\mu_{x}$ to $p$. If $x<p$, the argument is symmetric and the proposed change is to move mass $\mu_{x}$ to the highest signal $\hat{x}<x$ in the support of $\sigma$ or to zero if $x$ is the lowest signal in the support of $\sigma$. Hence, in what follows, we can restrict our attention to distributions in the set $\hat{\Sigma}$ of distributions such that $\widehat{\sigma} \in \Sigma$ (as defined by conditions (1) and (2)) and all positive signals in $\widehat{\sigma}$ are paired around some $p$ interpreted as the price.

Part 2. We now show that firms cannot attain a price strictly larger than $p^{*}$ with any distribution $\sigma \in \hat{\Sigma}$. Suppose all firms choose the same distribution $\sigma \in \hat{\Sigma}$ and consider a symmetric tie-breaking rule. Denote by $p$ the market clearing price. Suppose that $\sigma$ assigns positive mass to $2 n+1$ signals, $0, x_{1}^{-}, . ., x_{n}^{-}, x_{n}^{+}, . ., x_{1}^{+}$with $0 \leq x_{1}^{-}<. .<x_{n}^{-}<p<x_{n}^{+}<. .<x_{1}^{+}$and $x_{t}^{+}+x_{t}^{-}=2 p$ for all $t=1, . ., n$. Suppose there are also atomless parts of the distribution over the intervals $\left[a_{1}^{-}, b_{1}^{-}\right]$ and $\left[b_{1}^{+}, a_{1}^{+}\right] ; \ldots ;\left[a_{v}^{-}, b_{v}^{-}\right]$and $\left[b_{v}^{+}, a_{v}^{+}\right]$, where $0 \leq a_{1}^{-}<b_{1}^{-}<. .<a_{v}^{-}<b_{v}^{-}<p<$ $b_{v}^{+}<a_{v}^{+}<. .<b_{1}^{+}<a_{1}^{+}$and $a_{t}^{+}+a_{t}^{-}=b_{t}^{+}+b_{t}^{-}=2 p$ for all $t=1, . ., v$. In steps $1-4$, we show that one can induce a price $\hat{p} \geq p$ by using a distribution with at most two signals. In step 5, we show that no distribution with at most two signals induces a price higher than $p^{*}$, as defined in (7). We conclude that $p^{*}$ is the maximal market clearing price when firms choose a distribution $\sigma \in \hat{\Sigma}$.

Step 1. Consider signal $x_{n}^{-}, x_{n}^{+}$. Suppose $\mu_{x_{n}^{+}} \geq \mu_{x_{n}^{-}}$and $b_{n}^{-}<x_{n}^{-}$; that is, there is no atomless part of the distribution at a lower distance from the price (we consider the atomless parts of the distribution in step 3 below). Define as $\hat{X}$ the set of signals $x$ in the support of the distribution such that there exists a signal $2 p-x$ in the support of the distribution, that is

$$
\hat{X}=\left\{x \in \sigma: x \geq \min \left\{x_{1}^{-}, a_{1}^{-}\right\}\right\}
$$


and denote by $\mu_{0}$ the weight attached by $\sigma$ to signal 0 . Then one can induce a price $p+\Delta_{1}$, where $\Delta_{1} \geq 0$ will be defined below, by first moving $x_{n}^{+}$and $x_{n}^{-}$to $p$ and then moving all signals $x \in \hat{X}$ up by $\Delta_{1}$. To show this, we first show that by moving $x_{n}^{+}$and $x_{n}^{-}$to $p$ one can induce the same market clearing price $p$ and employ a signal distribution whose mean is lower than $\varphi$. Then, we can move all signals $x \in \hat{X}$ up by $\Delta_{1}$ to obtain a price $p+\Delta_{1}$ with a signal distribution in $\hat{\Sigma}$ whose average is $\varphi$.

Suppose firms assign weight $\mu_{x_{n}^{+}}+\mu_{x_{n}^{-}}$to signal $p$ instead of assigning weights $\mu_{x_{n}^{+}}$and $\mu_{x_{n}^{-}}$to signals $x_{n}^{+}$and $x_{n}^{-}$, respectively. Those who sample signal $p$ for all firms are indifferent between buying and selling. Denote by $\tau_{1}$ the fraction of them who buy. Suppose first that, before the change in the distribution, whenever an investor sampled signal $x_{n}^{+}$from firm $j$ and signal $x_{n}^{-}$from firm $\hat{\jmath}$ he bought stock $j$. The old aggregate demand is

$$
D_{1}=\sum_{y=1}^{F}\left(\begin{array}{l}
F \\
y
\end{array}\right)\left(\mu_{x_{n}^{+}}\right)^{y}\left(\mu_{x_{n}^{-}}\right)^{F-y}+Z_{1},
$$

where $Z_{1}$ depends on the signals further away from $p$ and is unaffected with the proposed change. The new aggregate demand (after the change) is

$$
\hat{D}_{1}=\tau_{1}\left(\mu_{x_{n}^{+}}+\mu_{x_{n}^{-}}\right)^{F}+Z_{1}
$$

Hence, one can define a $\tau_{1}<1$ such that $\hat{D}_{1}=D_{1}$ and so the market clears at $p$. Similarly, if an investor sampling signal $x_{n}^{+}$from firm $j$ and signal $x_{n}^{-}$from firm $\hat{\jmath}$ sold stock $\hat{\jmath}$, the old demand is $\tilde{D}_{1}=\left(\mu_{x_{n}^{+}}\right)^{F}+Z_{1}$ and there exists a $\tau_{1}<1$ such that $\hat{D}_{1}=D_{1}$. Notice that $\mu_{x_{n}^{+}} x_{n}^{+}+\mu_{x_{n}^{-}} x_{n}^{-} \geq\left(\mu_{x_{n}^{+}}+\mu_{x_{n}^{-}}\right) p$ since $\mu_{x_{n}^{+}} \geq \mu_{x_{n}^{-}}$and by definition $x_{n}^{-}=2 p-x_{n}^{+}$. Hence, we can define

$$
\Delta_{1}=\frac{1}{1-\mu_{0}}\left[\mu_{x_{n}^{+}} x_{n}^{+}+\mu_{x_{n}^{-}} x_{n}^{-}-\left(\mu_{x_{n}^{+}}+\mu_{x_{n}^{-}}\right) p\right],
$$

and move all signals $x \in \hat{X}$ up by $\Delta_{1}$ so as to satisfy condition (2) and have a price $p+\Delta_{1}$. The resulting distribution still belongs to $\hat{\Sigma}$. The same logic will be applied in the next steps.

Step 2. The procedure in step 1 can be repeated until one considers signals $x_{m}^{-}, x_{m}^{+}$where $m \equiv \max _{t}\left\{t: \mu_{x_{t}^{+}}<\mu_{x_{t}^{-}}\right\}$(if $\mu_{x_{n}^{+}}<\mu_{x_{n}^{-}}$, then $m=n$ ), or until one encounters an atomless part of the distribution at a lower distance from the price. Suppose one ends up with weight $\mu_{p_{2}}$ on signal $p_{2}$ and market clearing requiring that a fraction $\tau_{2}$ of those who sample signal $p_{2}$ for all firms buy. Consider first 
$x_{m}^{-}, x_{m}^{+}$. Following the same logic as in step 1 , one can move $x_{m}^{-}$to $x_{m-1}^{-}$and $x_{m}^{+}$ to $x_{m-1}^{+}$and then move all signals $x \in \hat{X}$ up by some $\Delta_{2} \geq 0$ so as to induce a price $p_{2}+\Delta_{2}$.

Consider the following weights for $x_{m-1}^{-}, x_{m-1}^{+}$and $p_{2}$, respectively: $\hat{\mu}_{x_{m-1}^{-}}=$ $\mu_{x_{m}^{-}}+\mu_{x_{m-1}^{-}}-k_{2}, \hat{\mu}_{x_{m-1}^{+}}=\mu_{x_{m}^{+}}+\mu_{x_{m-1}^{+}}-k_{2}$, and $\hat{\mu}_{p_{2}}=\mu_{p_{2}}+2 k_{2}$. Suppose a share $\tau_{m}$ of those who sample signal $p_{2}$ for all firms buy. We wish to define a $k_{2} \in\left(0, \mu_{x_{m}^{+}}\right)$and a $\tau_{m} \in(0,1)$ such that $p_{2}$ clears the market. Suppose first that whenever an investor samples signal $x_{m}^{+}$from firm $j$ and signal $x_{m}^{-}$from firm $\hat{\jmath}$ he buys stock $j$ and similarly for signals $x_{m-1} \cdot{ }^{27}$ The pre-change aggregate demand is

$$
\begin{aligned}
D_{2}=\sum_{y=1}^{F}\left(\begin{array}{c}
F \\
y
\end{array}\right)\left(\mu_{x_{m-1}^{+}}\right)^{y}\left(\mu_{x_{m-1}^{-}}+\mu_{x_{m}^{-}}+\mu_{x_{m}^{+}}+\mu_{p_{2}}\right)^{F-y}+ \\
\sum_{y=1}^{F}\left(\begin{array}{c}
F \\
y
\end{array}\right)\left(\mu_{x_{m}^{+}}\right)^{y}\left(\mu_{x_{m}^{-}}+\mu_{p_{2}}\right)^{F-y}+\tau_{2}\left(\mu_{p_{2}}\right)^{F}+Z_{2} .
\end{aligned}
$$

The new aggregate demand (considering the same symmetric tie-breaking rule after the change of distribution) is

$\hat{D}_{2}=\sum_{y=1}^{F}\left(\begin{array}{c}F \\ y\end{array}\right)\left(\mu_{x_{m-1}^{+}}+\mu_{x_{m}^{+}}-k_{2}\right)^{y}\left(\mu_{x_{m-1}^{-}}+\mu_{x_{m}^{-}}+\mu_{p_{2}}+k_{2}\right)^{F-y}+\tau_{m}\left(\mu_{p_{2}}+2 k_{2}\right)^{F}+Z_{2}$.

Using the binomial theorem and the convexity of $x \rightarrow x^{F}$ for $F \geq 2$, one can see that $\hat{D}_{2}>D_{2}$ when $k_{2}=0$ and $\tau_{m}=1$ and conversely $\hat{D}_{2}<D_{2}$ when $k_{2}=\mu_{x_{m}^{+}}$ and $\tau_{m}=0$. Hence, there exists a $k_{2} \in\left(0, \mu_{x_{m}^{+}}\right)$and a $\tau_{m} \in(0,1)$ such that $\hat{D}_{2}=D_{2}$. A similar argument can be applied in the case that, before the change, whenever an investor sampled signal $x_{m}^{+}$from firm $j$ and signal $x_{m}^{-}$from firm $\hat{\jmath}$ he sold stock $\hat{\jmath}$ and similarly for signals $x_{m-1}$. Now one can move all signals $x \in \hat{X}$ up by $\Delta_{2}$, where

$$
\begin{aligned}
\Delta_{2}=\frac{1}{1-\mu_{0}}[ & \mu_{x_{m}^{+}} x_{m}^{+}+\mu_{x_{m-1}^{+}} x_{m-1}^{+}+\mu_{x_{m}^{-}} x_{m}^{-}+\mu_{x_{m-1}^{-}} x_{m-1}^{-} \\
& \left.\quad-\left(\mu_{x_{m}^{+}}+\mu_{x_{m-1}^{+}}-k_{2}\right) x_{m-1}^{+}-\left(\mu_{x_{m}^{-}}+\mu_{x_{m-1}^{-}}-k_{2}\right) x_{m-1}^{-}-2 p_{2} k_{2}\right],
\end{aligned}
$$

and $\Delta_{2} \geq 0$ since $\mu_{x_{m}^{-}} \geq \mu_{x_{m}^{+}}$, so as to satisfy condition (2) and have a price $p_{2}+\Delta_{2}$.

\footnotetext{
${ }^{27}$ We can wlog assume the indifferences are broken in the same way when $x_{m}^{-}$vs $x_{m}^{+}$or $x_{m-1}^{-}$ vs $x_{m-1}^{+}$are drawn by satiating demand in one or the other.
} 
Step 3. Suppose one encounters an atomless part of the distribution and there is no other signal at a lower distance from the price. Suppose the price is $p_{3}$ and consider the distribution with density $g(x)$ over the interval $\left[a_{n}^{-}, b_{n}^{-}\right]$and density $h(x)$ over $\left[b_{n}^{+}, a_{n}^{+}\right]$. The logic of the previous steps can be applied by dividing the intervals $\left[a_{n}^{-}, b_{n}^{-}\right]$and $\left[b_{n}^{+}, a_{n}^{+}\right]$into sufficiently small subintervals.

Consider first the intervals $\left[b_{n}^{-}-\varepsilon, b_{n}^{-}\right]$and $\left[b_{n}^{+}, b_{n}^{+}+\varepsilon\right]$, where $\varepsilon$ is small. Define

$$
\mu^{+}=\int_{b_{n}^{+}}^{b_{n}^{+}+\varepsilon} h(x) d x, \text { and } x^{+}=\frac{1}{\mu^{+}} \int_{b_{n}^{+}}^{b_{n}^{+}+\varepsilon} x h(x) d x
$$

and similarly

$$
\mu^{-}=\int_{b_{n}^{-}-\varepsilon}^{b_{n}^{-}} g(x) d x, \text { and } x^{-}=\frac{1}{\mu^{-}} \int_{b_{n}^{-}-\varepsilon}^{b_{n}^{-}} x g(x) d x .
$$

If $\mu^{+}>\mu^{-}$and $\varepsilon \rightarrow 0$, one can obtain a larger price by moving all signals $x \in\left[b_{n}^{-}-\varepsilon, b_{n}^{-}\right] \cup\left[b_{n}^{+}, b_{n}^{+}+\varepsilon\right]$ to $p_{3}$ (following the logic of Step 1). If $\mu^{+}<\mu^{-}$ and $\varepsilon \rightarrow 0$, it is profitable to move all signals $x \in\left[b_{n}^{-}-\varepsilon, b_{n}^{-}\right]$to $b_{n}^{-}-\varepsilon$ and all $x \in\left[b_{n}^{+}, b_{n}^{+}+\varepsilon\right]$ to $b_{n}^{+}+\varepsilon$ (following the logic of Step 2). Finally, if $\mu^{+}=\mu^{-}$for all $\varepsilon \in\left[0, a_{n}^{+}-b_{n}^{+}\right]$, the same price $p_{3}$ can be obtained by moving all the mass $\mu^{+}$ into $x^{+}$and all the mass $\mu^{-}$into $x^{-}$.

Step 4. The argument in Steps 1-3 can be iterated until one obtains a distribution $0, x_{1}^{-}, p_{4}, x_{1}^{+}$, with $x_{1}^{+}=2 p_{4}-x_{1}^{-}$and $x_{1}^{-} \geq 0$ with weights $\mu_{0}, \mu_{x_{1}^{-}}, \mu_{p_{4}}, \mu_{x_{1}^{+}}$. Suppose $\mu_{x_{1}^{-}}<\mu_{x_{1}^{+}}$. Then one can increase the price by repeating the argument in step 1 and moving $x_{1}^{-}$and $x_{1}^{+}$to $p_{4}$. If $\mu_{0}=0$, we would end up with a onesignal distribution. If $\mu_{0}>0$, we would end up with a two-signals distribution with signals 0 and $\hat{p}_{4}$. Suppose instead $\mu_{x_{1}^{-}} \geq \mu_{x_{1}^{+}}$. Then one could increase the price by repeating the argument in step 2 and moving $x_{1}^{-}$to 0 and $x_{1}^{+}$to $2 p_{4}$. We would end up with a three-signals distribution with $0, \bar{p}, 2 \bar{p}$. Now consider the distribution $0, \bar{p}, 2 \bar{p}$, with weights respectively $\tilde{\mu}_{0}, \mu_{p}, \mu_{2 p}$. The aggregate supply is at least

$$
S_{4}=\sum_{y=1}^{F}\left(\begin{array}{l}
F \\
y
\end{array}\right)\left(\mu_{p}\right)^{F-y}\left(\tilde{\mu}_{0}\right)^{y}=\left(\tilde{\mu}_{0}+\mu_{p}\right)^{F}-\left(\mu_{p}\right)^{F} .
$$

We show that there exists a two-signals distribution inducing a larger price. Suppose a mass $k_{4}$ is moved from $\bar{p}$ to 0 and a mass $k_{4}$ is moved from $\bar{p}$ to $2 \bar{p}$. Condition 
(2) holds and there exists a tie breaking rule so that the new aggregate supply is

$$
\tilde{S}_{4}=\sum_{y=1}^{F}\left(\begin{array}{c}
F \\
y
\end{array}\right)\left(\tilde{\mu}_{0}+k_{4}\right)^{y}\left(\mu_{p}-2 k_{4}\right)^{F-y}=\left(\tilde{\mu}_{0}+\mu_{p}-k_{4}\right)^{F}-\left(\mu_{p}-2 k_{4}\right)^{F} .
$$

That induces a higher price if $\tilde{S}_{4}<S_{4}$, that is the case if $\tilde{S}_{4}$ decreases in $k_{4}$ at $k_{4}=0$. Taking the derivative of $\tilde{S}_{4}$ with respect to $k_{4}$, we need that

$$
\frac{d \tilde{S}_{4}}{d k_{4}}=(2)^{1 /(F-1)}\left(\mu_{p}-2 k_{4}\right)-\left(\tilde{\mu}_{0}+\mu_{p}-k_{4}\right)<0 .
$$

Notice that $d \tilde{S}_{4} / d k_{4}$ is decreasing in $k_{4}\left(1-2^{\frac{F}{F-1}}<0\right.$ for all $\left.F \geq 2\right)$, hence if $d \tilde{S}_{4} / d k_{4}<0$ at $k_{4}=0$ then it is negative everywhere. Hence, we need that

$$
(2)^{1 /(F-1)}\left(\mu_{p}\right) \leq\left(\tilde{\mu}_{0}+\mu_{p}\right)
$$

If condition (14) holds, setting $k_{4}=\mu_{p} / 2$ we obtain a two-signals distribution which induces a higher price. A similar argument can be applied if condition (14) is violated by moving a mass $\tilde{k}_{4}=\min \left\{\tilde{\mu}_{0}, \mu_{2 p}\right\}$ from 0 to $\bar{p}$ and from $2 \bar{p}$ to $\bar{p}$. Hence, the highest market clearing price is obtained with a two-signals distribution.

Step 5. We are then left with two-signals distributions which take one of the following forms: $\sigma_{a}=\left\{0,1-\mu_{a} ; 2 p_{a}, \mu_{a}\right\}$ or $\sigma_{b}=\left\{0,1-\mu_{b} ; p_{b}, \mu_{b}\right\}$ or $\sigma_{c}=$ $\left\{p_{c}, 1-\mu_{c} ; 2 p_{c}, \mu_{c}\right\}$. For the argument developed in the main text, among those distributions, the highest price is $p^{*}$, as defined in (7), and it is achieved by $\sigma^{*}$, as defined in (6). Q. E. D.

\section{Proof of Lemma 2}

We show that $\sigma^{*}$ and $p^{*}$, as defined respectively in (6) and (7), are part of an equilibrium. To simplify the notation, denote with $h$ the positive signal which is in the support of $\sigma^{*}$, that is $h=\varphi / \mu^{*}$, where $\mu^{*}$ is defined in (5). First, we show that there exists a tie-breaking rule such that $\left(\sigma^{*}, p^{*}\right)$ clears the market. Suppose that whenever indifferent between buying firm $r$ and selling another firm $j$ the investor buys $r$. Then since $p^{*}=h / 2$ only those who sample a signal 0 for all firms sell. The aggregate supply is $\left(1-\mu^{*}\right)^{F}$ that equals $1 / 2$. As the equilibrium is symmetric, that implies that the market clears for each firm.

Consider the possibility of deviations and suppose firm $j$ deviates and achieves a price $p^{j}>h / 2$. Let $p^{r}$ denote the price of non-deviating firm $r$, with $r \neq j$.

Step 1. We show that $p^{r} \leq h / 2$ for all firms $r \neq j$. 
The demand for non-deviating firm $r$ is at most

$$
D^{r} \leq \mu^{*} \prod_{w \neq r} \operatorname{Pr}\left(\left|p^{w}-x^{w}\right| \leq h-p^{r}\right)
$$

while the supply for $r$ is at least

$$
S^{r} \geq\left(1-\mu^{*}\right) \prod_{w \neq r} \operatorname{Pr}\left(\left|p^{w}-x^{w}\right|<p^{r}\right)
$$

Suppose by contradiction that $p^{r}>h / 2$. Since $\mu^{*}<1-\mu^{*}$ for all $F \geq 2$ and $\operatorname{Pr}\left(\left|p^{w}-x^{w}\right| \leq h-p^{r}\right) \leq \operatorname{Pr}\left(\left|p^{w}-x^{w}\right|<p^{r}\right)$, there is always excess supply for $r$. Hence, we must have $p^{r} \leq h / 2$ for all $r \neq j$.

We first assume that non-deviating firms are traded at the same price, and we denote this price by $p^{\prime}$ (see Step 5 for the case in which non-deviating firms are traded at different prices). In what follows, let $\mu_{1}$ denote the mass assigned by $\sigma^{j}$ to signals strictly below $p^{j}-p^{\prime}$, that is, $\mu_{1}=\operatorname{Pr}\left(x^{j}<p^{j}-p^{\prime}\right)$. Similarly, let $\mu_{2}=\operatorname{Pr}\left(p^{j}+p^{\prime} \leq x^{j}<p^{j}+h-p^{\prime}\right)$ and $\mu_{3}=\operatorname{Pr}\left(x^{j} \geq p^{j}+h-p^{\prime}\right)$.

Denote by $D^{j}$ and $S^{j}$ respectively the demand and supply for the deviating firm $j$, and similarly by $D^{-j}$ and $S^{-j}$ the demand and supply for non-deviating firms. Investors sell firm $j$ when they sample a signal $x^{j}<p^{j}-p^{\prime}$ together with signals 0 from $-j$, which occurs with probability $\left(1-\mu^{*}\right)^{F-1}$, and they demand $-j$ whenever a signal $h$ from $-j$ is sampled with a signal $x^{j}<p^{j}+h-p^{\prime}$ from firm $j$. Hence, we have

$$
S^{j}+D^{-j} \geq \mu_{1}\left(1-\mu^{*}\right)^{F-1}+\left(1-\left(1-\mu^{*}\right)^{F-1}\right)\left(1-\mu_{3}\right) .
$$

Investors demand $j$ at most when a signal $x^{j} \in\left[p^{j}, p^{j}+h\right)$ is sampled together with signals 0 from $-j$ or whenever a signal $x^{j} \geq p^{j}+h$ is sampled with any signal from $-j$, and they sell $-j$ whenever they sample signals 0 from firms $-j$ and a signal $x^{j} \in\left(p^{j}-p^{\prime}, p^{j}+p^{\prime}\right)$ from firm $j$. That is,

$$
D^{j}+S^{-j} \leq \mu_{3}+\left(1-\mu^{*}\right)^{F-1}\left(1-\mu_{1}-\mu_{3}\right)
$$

Since market clearing requires $S^{j}+D^{-j}=D^{j}+S^{-j}$ and $\left(1-\mu^{*}\right)^{F}=1 / 2$, we must have

$$
\mu_{1} \leq \mu^{*}+\mu_{3}\left(1-2 \mu^{*}\right)
$$

Step 2. We show that $p^{\prime}>0$. Suppose by contradiction $p^{\prime}=0$, then $\mu_{1}+\mu_{2}+\mu_{3}=$ 
1 and condition (15) writes as

$$
\mu_{3} \geq \frac{1-\mu_{2}-\mu^{*}}{2\left(1-\mu^{*}\right)}
$$

The average of firm $j$ 's distribution is at least $\mu_{2} p^{j}+\mu_{3}\left(h+p^{j}\right)$, which exceeds $\mu_{2} h / 2+3 \mu_{3} h / 2$ since we are assuming $p^{j}>h / 2$. Hence, given that $\mu^{*} h=\varphi$, condition (2) and (16) require

$$
\frac{\mu_{2}}{2}+\frac{1-\mu^{*}-\mu_{2}}{2\left(1-\mu^{*}\right)} \frac{3}{2}<\mu^{*}
$$

Since the left hand side of the above inequality decreases in $\mu_{2}$, the condition must be satisfied when $\mu_{2}$ is the largest, i.e. $\mu_{2}=1-\mu^{*}$ (as derived by letting $\mu_{3}=0$ ). The condition writes as $1-\mu^{*}<2 \mu^{*}$, which is violated for all $F \geq 2$. We conclude that we cannot have $p^{\prime}=0$.

Step 3. The aggregate supply of all firms is at least

$$
S^{j}+S^{-j} \geq\left(1-\mu_{2}-\mu_{3}\right)\left(1-\mu^{*}\right)^{F-1}
$$

as obtained when a signal 0 from all non-deviating firms is sampled with a signal $x^{j}<p^{j}+p^{\prime}$. Since market clearing requires $S^{j}+S^{-j}=1 / 2$, the previous condition requires

$$
\mu_{2}+\mu_{3} \geq \mu^{*}
$$

Step 4. The average of firm $j$ 's distribution is minimized when all signals $x^{j}<$ $p^{j}-p^{\prime}$ are concentrated at $x^{j}=0$, all signals $x^{j} \in\left[p^{j}+p^{\prime}, p^{j}+h-p^{\prime}\right)$ are concentrated at $x^{j}=p^{j}+p^{\prime}$, all signals $x^{j} \geq p^{j}+h-p^{\prime}$ are concentrated at $x^{j}=p^{j}+h-p^{\prime}$ and all other signals $x^{j} \in\left[p^{j}-p^{\prime}, p^{j}+p^{\prime}\right)$ are concentrated at $x^{j}=p^{j}-p^{\prime}$. That is, for condition (2) to hold, we need

$$
\mu_{2}\left(p^{j}+p^{\prime}\right)+\mu_{3}\left(p^{j}+h-p^{\prime}\right)+\left(1-\mu_{1}-\mu_{2}-\mu_{3}\right)\left(p^{j}-p^{\prime}\right) \leq \varphi
$$

Given (15) and (17), the left hand side is minimized when $\mu_{1}=\mu^{*}+\mu_{3}\left(1-2 \mu^{*}\right)$ and $\mu_{2}+\mu_{3}=\mu^{*}$. Substituting into (18), we have

$$
\left(\mu^{*}-\mu_{3}\right)\left(p^{j}+p^{\prime}\right)+\mu_{3}\left(p^{j}+h-p^{\prime}\right)+\left(1-\mu_{3}\right)\left(1-2 \mu^{*}\right)\left(p^{j}-p^{\prime}\right) \leq \varphi .
$$

The left hand side decreases in $p^{\prime}$ and so it is minimized when $p^{\prime}=h / 2$. Hence, $p^{j}>h / 2$ requires $\mu^{*} h<\varphi$, which is violated since by construction $\mu^{*} h=\varphi$. We 
conclude that there is no profitable deviation for firm $j$ when non-deviating firms are traded at the same price $p^{\prime}$.

Step 5. Suppose now $F>2$ and non-deviating firms are traded at a different price. Suppose two non-deviating firms, say firm 1 and firm 2, are traded respectively at prices $p_{1}$ and $p_{2}$. Assume wlog that $p_{1}<p_{2}$. When signal 0 from firm 1 is drawn together with signal 0 from firm 2 an investor prefers trading stock 2 (since $p_{1}<p_{2}$ ) and when signal 0 from firm 1 is drawn with signal $h$ from firm 2 an investor prefers trading stock 2 (since from Step $1 p_{1}<h-p_{2}$ ). Hence, $S_{1}=0$ and firm 1 is not traded. We are left with a market in which $F-1$ firms are traded. Suppose that, among them, the non-deviating firms are traded at the same price $p$. We can repeat the above argument and conclude that there is no profitable deviation. Suppose instead that among the $F-1$ traded firms there exist two non-deviating firms which are traded at a different price. We can repeat the above argument and end up with $F-2$ traded firms. Iterating, we end up with 2 traded firms, in which case we have already shown that there is no profitable deviation. We conclude that the profile $\left(\sigma^{*}, p^{*}\right)$ defines an equilibrium. Q. E. D.

\section{Proof of Proposition 6}

Suppose firms choose a distribution with support on $[0,2 \varphi]$, density $g$ symmetric around $\varphi$ and cdf $G$ such that $[1-2 G(x)]^{F-1}=1-x / \varphi$ for $x<\varphi$ and $[2 G(x)-1]^{F-1}=x / \varphi-1$ for $x \geq \varphi$.

Step 1. Market clearing requires $p=\varphi$. In fact, at $p=\varphi$, aggregate demand is

$$
D=F \int_{\varphi}^{2 \varphi} g(x)[2 G(x)-1]^{F-1} d x=F \frac{1}{\varphi} \int_{\varphi}^{2 \varphi} g(x)(x-\varphi) d x
$$

while aggregate supply is

$$
S=F \int_{0}^{\varphi} g(x)[1-2 G(x)]^{F-1} d x=F \frac{1}{\varphi} \int_{0}^{\varphi} g(x)(\varphi-x) d x .
$$

Since $\int_{0}^{2 \varphi} g(x) d x=1$ and $\int_{0}^{2 \varphi} x g(x) d x=\varphi$ due to condition $(2)$, we have $D=S$. That is, the market clears.

Step 2. There is no profitable deviation. To see this, suppose firm $j$ deviates to a distribution $H$ with density $h$. Denote with $p^{j}$ the price of $j$ and with $p$ the price of non-deviating firms. Notice first that market clearing requires $p=\varphi$. In 
fact, if $p=\varphi$ aggregate demand for non-deviating firms is

$$
D^{-j}=(F-1) \int_{\varphi}^{2 \varphi} g(x)[2 G(x)-1]^{F-2}\left[H\left(p^{j}+x-\varphi\right)-H\left(\varphi+p^{j}-x\right)\right] d x,
$$

while aggregate supply is

$$
S^{-j}=(F-1) \int_{0}^{\varphi} g(x)[1-2 G(x)]^{F-2}\left[H\left(p^{j}+\varphi-x\right)-H\left(p^{j}+x-\varphi\right)\right] d x .
$$

By symmetry of $g$, for any $x \leq \varphi$ there exists a signal $v=2 \varphi-x$ such that $g(x)=$ $g(v)$. Hence, $G(x)=1-G(v), H(x-\varphi)=H(\varphi-v)$ and $H(\varphi-x)=H(v-\varphi)$, which imply $D^{-j}=S^{-j}$ for $p=\varphi$. To see that $p=\varphi$ is the only market clearing price for non-deviating firms, suppose $p>\varphi$. Then the new aggregate demand is

$$
\hat{D}^{-j}=(F-1) \int_{p}^{2 \varphi} g(x)[G(x)-G(2 p-x)]^{F-2}\left[H\left(p^{j}+x-p\right)-H\left(p+p^{j}-x\right)\right] d x .
$$

Notice that $p>\varphi$ implies $G(x)-G(2 p-x)<G(x)-G(2 \varphi-x)$ and $H\left(p^{j}+x-\right.$ $p)-H\left(p+p^{j}-x\right)>H\left(p^{j}+x-\varphi\right)-H\left(\varphi+p^{j}-x\right)$, so it must be that $\hat{D}^{-j}<D^{-j}$. Similarly, the new aggregate supply is $\hat{S}^{-j}>S^{-j}$. Hence, there is excess supply and so $p>\varphi$ does not clear the market. The argument which rules out $p<\varphi$ is symmetric. Suppose then $p^{j}=\varphi$. The demand for $j$ is

$$
D^{j}=\int_{\varphi}^{\infty} h(x)[2 G(x)-1]^{F-1} d x=\frac{1}{\varphi} \int_{\varphi}^{2 \varphi} h(x)(x-\varphi) d x+\int_{2 \varphi}^{\infty} h(x) d x
$$

while the supply of $j$ is

$$
S^{j}=\int_{0}^{\varphi} h(x)[1-2 G(x)]^{F-1} d x=\frac{1}{\varphi} \int_{0}^{\varphi} h(x)(\varphi-x) d x .
$$

Since $D^{j} \leq S^{j}$ at $p^{j}=\varphi$, it must be that $p^{j} \leq \varphi$. Hence, there is no profitable deviation. Q. E. D.

\section{Proof of Proposition 7}

Suppose first that $f(\cdot)$ is strictly concave in gains from trade. Suppose the firm chooses the distribution $\{\varphi-\varepsilon-\delta, 1 / 2-\nu ; \varphi+\varepsilon, 1 / 2+\nu\}$, where $\varepsilon$ and $\delta$ 
are positive and $\nu=\delta /(4 \varepsilon+2 \delta)$ due to condition (2). At $p=\varphi$, the demand is $(1 / 2+\nu) f(\varepsilon)$ while the supply is $(1 / 2-\nu) f(\varepsilon+\delta)$. Since $(1 / 2+\nu) /(1 / 2-$ $\nu)=(\delta+\varepsilon) / \varepsilon$ and by concavity of $f(\cdot)$ that exceeds $f(\varepsilon+\delta) / f(\varepsilon)$, we have excess demand at $p=\varphi$. That is, market clearing requires $p>\varphi$. If $f(\cdot)$ is strictly convex in gains from trade, the same argument applies considering the distribution $\{\varphi-\varepsilon+\delta, 1 / 2+w ; \varphi+\varepsilon, 1 / 2-w\}$, where $\varepsilon$ and $\delta$ are positive and $w=\delta /(4 \varepsilon-2 \delta)$. Q. E. D.

\section{Proof of Proposition 8}

We show that $\sigma^{*}$ and $p^{*}$, as defined respectively in (6) and (7), are part of an equilibrium. To simplify the notation, denote by $h$ the positive signal which is in the support of $\sigma^{*}$, that is $h=\varphi / \mu^{*}$, where $\mu^{*}$ is defined in (5). Consider the possibility of deviations and suppose firm $j$ deviates and achieves a price $p^{j}>h / 2$. Let $p^{r}$ denote the price of non-deviating firm $r$, with $r \neq j$.

Step 1. We must have that $p^{r} \leq h / 2$ for all firms $r \neq j$. The proof is the same as in Step 1 of the proof of Lemma 2.

We first assume that non-deviating firms are traded at the same price, and we denote this price by $p^{\prime}$ (see Step 4 for the case in which non-deviating firms are traded at different prices). In what follows, let $\mu_{3}$ denote the mass assigned by $\sigma^{j}$ to signals strictly above $p^{j}+p^{\prime}$, that is, $\mu_{3}=\operatorname{Pr}\left(x^{j}>p^{j}+p^{\prime}\right)$ and similarly $\mu_{2}=\operatorname{Pr}\left(x^{j}=p^{j}+p^{\prime}\right)$. Denote by $D^{j}$ and $S^{j}$ respectively the demand and supply for the deviating firm $j$, and similarly by $D^{-j}$ and $S^{-j}$ the demand and supply for non-deviating firms.

Step 2. Suppose first that $p^{\prime}=h / 2$. In order to have $p^{j}>h / 2$, there must be excess net aggregate demand at $p^{j}=h / 2$. In order to show that this is not possible, we first define the maximal net aggregate demand. The aggregate supply includes at least the draws of a signal 0 from all non-deviating together with a signal $x^{j}<p^{j}+p^{\prime}$ from the deviating firm. That is, we have

$$
S^{j}+S^{-j} \geq\left(1-\mu^{*}\right)^{F-1}\left(1-\mu_{2}-\mu_{3}\right) f\left(\frac{h}{2}\right) .
$$

The aggregate demand includes at most all other cases. Moreover, notice that we can collapse all signals $x^{j}>p^{j}+p^{\prime}$ into their average value $x_{3}$. That would not affect the demand of non-deviating firms nor the aggregation constraint (2), and given the concavity of $f()$ that would not decrease the demand of the deviating

firm. Hence, we have

$$
D^{j}+D^{-j} \leq \mu_{3} f\left(x_{3}-\frac{h}{2}\right)+\mu_{2} f\left(\frac{h}{2}\right)+\left(1-\left(1-\mu^{*}\right)^{F-1}\right)\left(1-\mu_{2}-\mu_{3}\right) f\left(\frac{h}{2}\right) .
$$


From the aggregation constraint (2), we have

$$
x_{3}<\frac{\mu^{*}-\mu_{2}}{\mu_{3}} h .
$$

Substituting (21) into (20), and recalling that $\left(1-\mu^{*}\right)^{F}=1 / 2$, we have that $p^{j}>h / 2$ requires

$$
\mu_{3} f\left(\frac{\mu-\mu_{2}}{\mu_{3}} h-\frac{h}{2}\right)+\mu_{2} f\left(\frac{h}{2}\right)>\frac{\mu^{*}}{1-\mu^{*}}\left(1-\mu_{2}-\mu_{3}\right) f\left(\frac{h}{2}\right) .
$$

Consider now that $f(x)=x^{z}$. For convenience of notation, define

$$
y=\frac{2\left(\mu^{*}-\mu_{2}\right)}{\mu_{3}}-1
$$

and notice that $x_{3}>h$ requires $y>1$. Condition (22) can be written as

$$
\mu_{3}\left(y \frac{h}{2}\right)^{z}+\left(\mu^{*}-\frac{1+y}{2} \mu_{3}\right)\left(\frac{h}{2}\right)^{z}>\frac{\mu^{*}}{1-\mu^{*}}\left(1-\left(\mu^{*}-\frac{1+y}{2} \mu_{3}\right)-\mu_{3}\right)\left(\frac{h}{2}\right)^{z},
$$

that is

$$
\left(\frac{h}{2}\right)^{z} \frac{\mu_{3}}{1-\mu^{*}}\left((y)^{z}\left(1-\mu^{*}\right)-\frac{1}{2} y+\mu^{*}-\frac{1}{2}\right)>0 .
$$

Define $h(y)=(y)^{z}\left(1-\mu^{*}\right)-\frac{1}{2} y+\mu^{*}-\frac{1}{2}$ and notice that $h(1)=0$ and $h^{\prime}(y)<0$ when $z\left(1-\mu^{*}\right)<1 / 2$ (in fact, $h^{\prime \prime}(y)<0$ for all $z<1$ and $h^{\prime}(1)<0$ when $\left.z\left(1-\mu^{*}\right)<1 / 2\right)$. Hence $h(y)<0$ for all $y>1$ and that contradicts condition (22). That is, we cannot have $p^{j}>h / 2$ when $p^{\prime}=h / 2$.

Step 3. We now show that decreasing $p^{\prime}$ cannot increase $p^{j}$. Suppose we decrease $p^{\prime}$, say from $\tilde{p}$ to $\tilde{p}-\varepsilon$, and consider the effects on the net demand for stock $j$. Denote with $\mu_{1}$ the mass of signals at $p^{j}-\tilde{p}$, that is $\mu_{1}=\operatorname{Pr}\left(x^{j}=p^{j}-\tilde{p}\right)$. Investors who sample $x^{j}=p^{j}-\tilde{p}$ together with signal 0 from all other firms now strictly prefer selling firm $j$. Hence, the supply $S^{j}$ increases by $\mu_{1}\left(1-\mu^{*}\right)^{F-1}\left(1-\frac{1}{F}\right)$. Consider the effects on the demand $D^{j}$. If $\sigma^{j}$ assigns positive mass to $x^{j}=h+p^{j}-\tilde{p}$, investors who sample $x^{j}=h+p^{j}-\tilde{p}$ with a signal $h$ from any other firm $-j$ now strictly prefer not to buy firm $j$. That pushes the demand $D^{j}$ down. If $\sigma^{j}$ assigns positive mass to $x^{j}=p^{j}+\tilde{p}$, investors who sample $x^{j}=p^{j}+\tilde{p}$ with signal 0 from all other firms $-j$ now strictly prefer to buy firm $j$. However, that does not affect the demand $D^{j}$ since the tie-breaking rule supporting the equilibrium in (6) and (7) already assigned all the demand to firm $j$. Finally, notice that it is never optimal that $\sigma^{j}$ assigns positive mass to signals in $\left(p^{j}, p^{j}+\tilde{p}\right)$. Those signals 
can be decreased to $p^{j}$ as that would not affect the demand of any firm while at the same time allowing to increase all the other signals in $\sigma^{j}$ and satisfy the aggregation condition (2). Hence, decreasing $p^{\prime}$ involves no change in the demand due to signals slightly below $p^{j}+\tilde{p}$. Hence, the demand $D^{j}$ cannot increase as we decrease $p^{\prime}$. It follows that $D^{j}-S^{j}$ cannot increase and, given that the price $p^{j}$ is a smooth function of the net demand $D^{j}-S^{j}, p^{j}$ cannot increase as we decrease $p^{\prime}$. That shows that we cannot have $p^{j}>h / 2$ when $p^{\prime} \leq h / 2$.

Step 4. Suppose now $F>2$ and non-deviating firms are traded at a different price. The argument to rule out that this cannot help the deviating firm is the same as in Step 5 of the proof of Lemma 2. We conclude that the profile $\left(\sigma^{*}, p^{*}\right)$ is part of an equilibrium. Q. E. D.

\section{Proof of Proposition 9}

Suppose that $X^{j} \in[0, H]$ and let $\mu^{*}=1-(1 / 2)^{1 / F}$. If $\varphi / \mu^{*} \leq H$, the upper bound does not bind and the analysis of Proposition 2 applies. Suppose instead $\varphi /\left(1-\mu^{*}\right) \geq H$. In this case, one can obtain a price $p=H$ with the distribution $\sigma=\{0,1-\eta ; H, \eta\}$ in which $(\eta)^{F} \geq 1 / 2$, that is $\eta \geq 1-\mu^{*}$. Since $\varphi \geq\left(1-\mu^{*}\right) H$, condition (2) is satisfied. This is obviously the highest price irrespective of $F$. Hence, in what follows, we focus on

$$
H \in\left(\frac{\varphi}{1-\mu^{*}}, \frac{\varphi}{\mu^{*}}\right)
$$

We first show that if $H \geq \tilde{H}$, as defined by condition (10), the highest market clearing price is achieved with $\sigma_{H}=\left\{\left(\varphi-\mu^{*} H\right) /\left(1-\mu^{*}\right), 1-\mu^{*} ; H, \mu^{*}\right\}$ and it is defined as in (11) by

$$
p_{H}=\frac{1}{2}\left(H+\frac{\varphi-\mu^{*} H}{1-\mu^{*}}\right) .
$$

By the same argument a the one developed in Lemma 1, the highest market clearing price is obtained when the distribution takes one of the following forms. Either, $\sigma_{a}=\left\{0,1-\mu_{a} ; p_{a}, \mu_{a}\right\}$ with $\left(\mu_{a}\right)^{F} \geq 1 / 2$ and so the highest price is obtained when $\mu_{a}=1-\mu^{*}$ and it writes as

$$
p_{a}=\frac{\varphi}{1-\mu^{*}} .
$$

The price defined in (24) exceeds $p_{a}$ in $(25)$ if

$$
H \geq \frac{\varphi}{1-2 \mu^{*}}
$$


Or $\sigma_{b}=\left\{0, \tilde{\mu}, l, 1-\mu_{b}-\tilde{\mu} ; H, \mu_{b}\right\}$ with $p_{b}=(H+l) / 2$ and because of market clearing

$$
1-(1-\tilde{\mu})^{F}+\left(1-\mu_{b}-\tilde{\mu}\right)^{F}=1 / 2 .
$$

Differently from Lemma 1 , it may be optimal to have $\tilde{\mu}>0$ since shifting signals $l$ and $H$ further away from the price is not feasible. Since $\sigma_{H}$ is obtained as a special case of $\sigma_{b}$ when $\tilde{\mu}=0$, we investigate under which condition $p_{b}$ is maximized by $\tilde{\mu}=0$. Given the market clearing condition (27), $l$ is defined by condition (2) and so we have

$$
p_{b}=\frac{1}{2}\left(H+\frac{\varphi-\left(1-\tilde{\mu}-\left((1-\tilde{\mu})^{F}-\frac{1}{2}\right)^{\frac{1}{F}}\right) H}{\left((1-\tilde{\mu})^{F}-\frac{1}{2}\right)^{\frac{1}{F}}}\right) .
$$

Differentiating $p_{b}$ with respect to $\tilde{\mu}$, we see that $p_{b}$ decreases in $\tilde{\mu}$ if

$$
2 \varphi-H \leq 0
$$

That is, under condition (29), $p_{b}$ is maximized by $\tilde{\mu}=0$. Hence, if conditions (26) and (29) are satisfied, as required by condition (10) in the text, the highest market clearing price is defined by (24). This price increases in $F$ since $\mu^{*}$ decreases in $F$.

We now show that $p_{H}$ can be sustained in equilibrium. The logic follows closely the proof of Lemma 2. Suppose firm $j$ deviates and achieves a price $p^{j}>(H+l) / 2$ and let $p^{r}$ denote the price of non-deviating firm $r$, with $r \neq j$.

Step 1. Following the same argument as the one in Step 1 of the proof of Lemma 2, we must have $p^{r} \leq(H+l) / 2$ for all $r \neq j$ or there would be excess supply for firm $r$.

We first assume that non-deviating firms are traded at the same price, and we denote it with $p^{\prime}$ (see Step 5 for the case in which non-deviating firms are traded at different prices). Let $\mu_{0}=\operatorname{Pr}\left(x^{j}<p^{j}+p^{\prime}-H\right) ; \mu_{1}=\operatorname{Pr}\left(p^{j}+p^{\prime}-H \leq x^{j}<\right.$ $\left.p^{j}-p^{\prime}+l\right)$, and $\mu_{2}=\operatorname{Pr}\left(p^{j}+p^{\prime}-l \leq x^{j} \leq H\right)$.

Following the logic of Lemma 2, we have that market clearing requires

$$
\mu_{0}+\mu_{1} \leq \mu^{*}
$$

Step 2. We show that $p^{\prime}>l$. Suppose by contradiction that $p^{\prime}=l$, then $\mu_{0}+\mu_{1}+\mu_{2}=1$ and (30) writes as

$$
\mu_{2} \geq 1-\mu^{*}
$$

The average of firm $j$ 's distribution is at least $\mu_{2} p^{j}$, and so given (31) and $p^{j}>$ 
$(H+l) / 2$, condition $(2)$ requires

$$
\left(1-\mu^{*}\right)(H+l)<2 \varphi
$$

that is, $H+\varphi-2 H \mu^{*}<2 \varphi$, and that violates (26). We conclude that we cannot have $p=l$.

Step 3. Following the logic of Step 3 in the proof of Lemma 2, we need

$$
\mu_{2} \geq \mu^{*}+\mu_{0}\left(1-2 \mu^{*}\right)
$$

or we would have excess aggregate supply.

Step 4. Condition (2) requires

$$
\mu_{1}\left(p^{j}+p^{\prime}-H\right)+\mu_{2}\left(p^{j}+p^{\prime}-l\right)+\left(1-\mu_{0}-\mu_{1}-\mu_{2}\right)\left(p^{j}-p^{\prime}+l\right) \leq \varphi .
$$

The previous expression is linear in $p^{\prime}$, so it must hold either for $p^{\prime}=(H+l) / 2$ or for $p^{\prime} \rightarrow l$. Suppose $p^{\prime}=(H+l) / 2$, we must have $\mu_{2} H+\left(1-\mu_{0}-\mu_{2}\right) l<\varphi$, which given (30) and (32) must hold when $\mu_{0}=\mu^{*}-\mu_{1}$ and $\mu_{2}=\mu^{*}+\left(\mu^{*}-\mu_{1}\right)\left(1-2 \mu^{*}\right)$. That is, we need $\left(\mu^{*}-\mu_{1}\right)\left(H-2 l-2 H \mu^{*}+2 l \mu^{*}\right)<0$, which is equivalent to $H<2 \varphi$, and that violates condition (29). Suppose instead $p^{\prime} \rightarrow l$, (33) requires $\left(1-\mu_{0}-\mu_{1}\right)(H+l)<2 \varphi$, which given $(30)$ requires $\left(1-\mu^{*}\right)(H+l)<2 \varphi$. As shown in Step 2, this violates (26).

Step 5. The argument to show that there are no profitable deviations when some non-deviating firms are traded at a different price follows Step 5 of the proof of Lemma 2. Q. E. D.

\section{Proof of Proposition 10}

Suppose firm $j$ has fundamental $\varphi^{j}=\varphi+\varepsilon^{j}, \sigma^{j}=\left\{\varepsilon^{j}, 1-\mu^{*} ; 2 p^{*}+\varepsilon^{j}, \mu^{*}\right\}$ and prices are $p^{j}=p^{*}+\varepsilon^{j}$. The logic to show that the market clears is the same as in Lemma 2. Let $h=\varphi / \mu^{*}$, where $\mu^{*}=1-(1 / 2)^{1 / F}$, and suppose firm $j$ deviates and achieves a price $p^{j}>h / 2+\varepsilon^{j}$. Let $p^{r}$ denote the price of non-deviating firm $r$, with $r \neq j$.

Step 1. Following the argument of Step 1 in the proof of Lemma 2, we establish that $p^{r} \leq h / 2+\varepsilon^{r}$ for all $r \neq j$ as otherwise there would be excess supply for firm $r$.

Assume first that the price of the non-deviating firms takes the following form:

$$
p^{r}=\varepsilon^{r}+\lambda h \text { for all } r \neq j,
$$


where, due to Step $1, \lambda \leq 1 / 2$. In Step 5 , we consider the general case in which $p^{r}=\varepsilon^{r}+\lambda^{r} h$. Let $\mu_{0}=\operatorname{Pr}\left(x^{j}<p^{j}-(1-\lambda) h\right) ; \mu_{1}=\operatorname{Pr}\left(p^{j}-(1-\lambda) h \leq x^{j}<p^{j}-\lambda h\right) ;$ $\mu_{2}=\operatorname{Pr}\left(p^{j}+\lambda h \leq x^{j}<p^{j}+(1-\lambda) h\right)$; and $\mu_{3}=\operatorname{Pr}\left(x^{j} \geq p^{j}+(1-\lambda) h\right)$.

Following the logic of Lemma 2, we have that market clearing requires

$$
\mu_{0}+\mu_{1} \leq \mu^{*}+\mu_{3}\left(1-2 \mu^{*}\right)
$$

Step 2. We show that $\lambda>0$ when $F>2$.

Suppose by contradiction that $\lambda=0$ and so $\mu_{0}+\mu_{1}+\mu_{2}+\mu_{3}=1$. Hence, (34) writes as

$$
\mu_{3} \geq \frac{1-\mu_{2}-\mu^{*}}{2\left(1-\mu^{*}\right)}
$$

The average of firm $j$ 's distribution is at least $\mu_{1}\left(p^{j}-h\right)+\mu_{2} p^{j}+\mu_{3}\left(h+p^{j}\right)$, which exceeds $\mu_{1}\left(\varepsilon^{j}-h / 2\right)+\mu_{2}\left(h / 2+\varepsilon^{j}\right)+\mu_{3}\left(3 h / 2+\varepsilon^{j}\right)$ since we are assuming $p^{j}>h / 2+\varepsilon^{j}$. Suppose first $\varepsilon^{j}<h / 2$. Conditions (2) and (35) require

$$
\mu_{2}\left(\frac{h}{2}+\varepsilon^{j}\right)+\left(\frac{1-\mu_{2}-\mu^{*}}{2\left(1-\mu^{*}\right)}\right)\left(\frac{3 h}{2}+\varepsilon^{j}\right)<\varphi+\varepsilon^{j} .
$$

Since the left hand side of the above inequality decreases in $\mu_{2}$, it must be satisfied when $\mu_{2}$ is the largest. Substituting $\mu_{2}=1-\mu^{*}$ and $\varphi=\mu^{*} h$ into (36), we have $h\left(1-3 \mu^{*}\right)<2 \mu^{*} \varepsilon^{j}$, which is violated for all $F>2$. A similar argument applies when $\varepsilon^{j} \geq h / 2$ by noticing that the average of firm $j$ 's distribution is minimized when $\mu_{2}=1-\mu^{*}$ and so in particular we must have $\left(1-\mu^{*}\right) h / 2<\mu^{*}\left(h+\varepsilon^{j}\right)$. Given (12), that requires $\left(1-\mu^{*}\right) h / 2<\mu^{*}\left(h+\left(1-2 \mu^{*}\right) h\right)$, which is violated for any $F>2$. We conclude that we cannot have $\lambda=0$ when $F>2$.

Step 3. Suppose $\lambda>0$. The aggregate supply of all firms is at least

$$
S^{j}+S^{-j} \geq \mu_{0}+\left(1-\mu_{2}-\mu_{3}-\mu_{0}\right)\left(1-\mu^{*}\right)^{F-1},
$$

as obtained when a signal $x^{j}<p^{j}-(1-\lambda) h$ from firm $j$ is sampled with any other signal from firms $-j$ and when a signal $\varepsilon^{r}$ from all firms $r \neq j$ is sampled with a signal $x^{j}<p^{j}+\lambda h$ from firm $j$. Hence, market clearing requires

$$
\mu_{2}+\mu_{3} \geq \mu^{*}+\mu_{0}\left(1-2 \mu^{*}\right)
$$


Step 4. Condition (2) requires

$$
\begin{aligned}
\mu_{1}\left(p^{j}-(1-\lambda) h\right)+\mu_{2}\left(p^{j}+\lambda h\right) & +\mu_{3}\left(p^{j}+(1-\lambda) h\right)+ \\
& \left(1-\mu_{0}-\mu_{1}-\mu_{2}-\mu_{3}\right)\left(p^{j}-\lambda h\right) \leq \varphi+\varepsilon^{j} .
\end{aligned}
$$

Suppose first $F>2$ and so $\lambda>0$. Given (34) and (37), the left hand side is minimized when $\mu_{1}=\mu^{*}+\mu_{3}\left(1-2 \mu^{*}\right)-\mu_{0}$ and $\mu_{2}=\mu^{*}+\mu_{0}\left(1-2 \mu^{*}\right)-\mu_{3}$. Substituting into (38), we see that the left hand side of (38) decreases in $\lambda$ and so it must hold when $\lambda=1 / 2$. This in turn requires $\varepsilon^{j}>\left(1-2 \mu^{*}\right) h$, which violates condition (12) in the text.

Suppose finally that $F=2$. The left hand side of inequality (38) is linear in $\lambda$, so it must hold either for $\lambda=1 / 2$ or for $\lambda=0$. If $\lambda=1 / 2$, we can repeat the argument above and conclude that $\varepsilon^{j}>\left(1-2 \mu^{*}\right) h$ violates condition (12) in the text (notice that $2\left(p^{*}-\varphi\right)=\varphi \sqrt{2}$ when $F=2$ and so $\varepsilon^{j} \leq \varphi \sqrt{2} / 2$ implies $\left.\varepsilon^{j} \leq 2\left(p^{*}-\varphi\right)\right)$. Suppose then $\lambda=0$. Condition (38) requires in particular $\mu_{2} p^{j}+\mu_{3}\left(p^{j}+h\right) \leq \varphi+\varepsilon^{j}$, which given (35) requires

$$
\mu_{2} p^{j}+\frac{1-\mu_{2}-\mu^{*}}{2\left(1-\mu^{*}\right)}\left(p^{j}+h\right) \leq \varphi+\varepsilon^{j}
$$

The left hand side is linear in $\mu_{2}$ and so it must hold when either $\mu_{2}=0$ or $\mu_{2}=1-\mu^{*}$. Suppose $\mu_{2}=0$, we have $p^{j} \leq 2 \varepsilon^{j}-\left(1-2 \mu^{*}\right) h$, that implies $p^{j}<$ $h / 2+\varepsilon^{j}$ since by condition (12) $\varepsilon^{j} \leq \varphi \sqrt{2} / 2$. Suppose then $\mu_{2}=1-\mu^{*}$, we have $p^{j} \leq\left(\varphi+\varepsilon^{j}\right) /\left(1-\mu^{*}\right)$. Condition (12) is equivalent to $\left(\varphi+\varepsilon^{j}\right) /\left(1-\mu^{*}\right) \leq h / 2+\varepsilon^{j}$. We conclude that there is no profitable deviation for firm $j$ when non-deviating firms are traded at prices $p^{r}=\varepsilon^{r}+\lambda h$.

Step 5. Suppose each non-deviating firm is traded at a price $p^{r}=\varepsilon^{r}+\lambda^{r} h$. The argument to show that there are no profitable deviations follows Step 5 of the proof of Lemma 2. Q. E. D. 